\title{
Two estimators of the long-run variance: beyond short memory
}

\author{
Karim M. Abadir* \\ Imperial College Business School, Imperial College London, London SW7 2AZ, UK \\ Walter Distaso \\ Imperial College Business School, Imperial College London, London SW7 2AZ, UK \\ Liudas Giraitis \\ Department of Economics, Queen Mary, University of London, London E14 NS, UK
}

January 19, 2009

\begin{abstract}
This paper deals with the estimation of the long-run variance of a stationary sequence. We extend the usual Bartlett-kernel heteroskedasticity and autocorrelation consistent (HAC) estimator to deal with long memory and antipersistence. We then derive asymptotic expansions for this estimator and the memory and autocorrelation consistent (MAC) estimator introduced by Robinson (2005). We offer a theoretical explanation for the sensitivity of HAC to the bandwidth choice, a feature which has been observed in the special case of short memory. Using these analytical results, we determine the MSE-optimal bandwidth rates for each estimator. We analyze by simulations the finite-sample performance of $\mathrm{HAC}$ and MAC estimators, and the coverage probabilities for the studentized sample mean, giving practical recommendations for the choice of bandwidths.
\end{abstract}

JEL Classification: C22, C14.

Keywords: long-run variance, long memory, heteroskedasticity and autocorrelation consistent (HAC) estimator, memory and autocorrelation consistent (MAC) estimator.

${ }^{*}$ Corresponding author. E-mail address: k.m.abadir@imperial.ac.uk. 


\section{Introduction and setup}

In empirical studies, it is now standard practice to produce robust estimates of standard errors (SEs). Popular references in econometrics for such procedures include White (1980), Newey and West (1987), Andrews and Monahan (1992). In statistics, the literature goes further back to Jowett (1955) and Hannan (1957). These procedures for estimating covariance matrices account for heteroskedasticity and autocorrelation of unknown form, for short memory models.

There is now an increasing body of evidence suggesting the existence of long memory in macroeconomic and financial series; e.g. see Diebold and Rudebusch (1989), Baillie and Bollerslev (1994), Gil-Alaña and Robinson (1997), Chambers (1998), Cavaliere (2001), Abadir and Talmain (2002). It is therefore of interest to adapt the most popular of these procedures, the Bartlett-kernel heteroskedasticity and autocorrelation consistent (HAC) estimator, to account for the possibility of long memory and antipersistence. In addition to HAC, we study the alternative memory and autocorrelation consistent (MAC) estimator recently introduced by Robinson (2005). He established the consistency of his MAC estimator of the covariance matrix, leaving open the issue of its higher-order expansion.

Our first contribution is to derive second order expansions for HAC and MAC in the univariate case, reducing the problem to the estimation of a scalar (the long run variance) instead of estimating the covariance matrix. Our derivations give an insight into the more difficult multivariate case and provide the first step in understanding this problem.

The second contribution of this paper is to provide a theoretical explanation for the sensitivity of HAC estimators to the choice of bandwidth, a feature that has been widely observed in the special case of short memory. Our results show that the HAC estimator is sensitive because the minimum-MSE bandwidth depends on the persistence in the series. The theoretical part of this paper explains where the problem comes from and gives some practical advice for selecting the bandwidth. We also show that, on the other hand, the MAC estimator is more robust to the bandwidth selection, since its asymptotic properties are not affected by long memory or antipersistence.

The final theoretical contribution of this paper is to obtain the distribution of the estimated normalized spectrum at the origin, by virtue of its link to the long-run variance. The distribution is Gaussian for MAC, but the one for HAC is 
Gaussian only if the long memory is below some threshold. In the case of short memory, HAC is the usual Bartlett-kernel estimator of the spectral density at zero frequency, and its bias and asymptotic distribution are well investigated in the literature. The asymptotic results for the HAC estimator provide the background for the development of kernel estimation of a spectral density under long memory and antipersistence.

The plan of the paper is as follows. In Sections 2 and 3, we derive the bias and asymptotic expansions for both types of estimators, allowing us to describe the limiting distributions as well as the asymptotic MSEs. This enables us to determine the rate of the MSE-optimal bandwidth for each estimator. Section 4 investigates by simulations the finite-sample performance of HAC and MAC estimators, and coverage probabilities for the studentized sample mean, giving practical recommendations for the choice of bandwidths. Section 5 concludes. The derivations are given in the Appendix.

We now detail the setting for our paper. Let $\left\{X_{t}\right\}_{t \in \mathbb{Z}}$ be a stationary sequence with unknown mean $\mu:=\mathrm{E}\left(X_{t}\right)$. Let the spectral density of $\left\{X_{t}\right\}$ be denoted by $f(\lambda)$ and defined over $|\lambda| \leq \pi$. Suppose that it has the property

$$
f(\lambda)=b_{0}|\lambda|^{-2 d}+o\left(|\lambda|^{-2 d}\right), \text { as } \lambda \rightarrow 0,
$$

where $|d|<1 / 2$ and $b_{0}>0$. Special cases include stationary and invertible $\operatorname{ARIMA}(p, d, q)$ : when $p$ and $q$ are finite; but see Abadir and Taylor (1999) for identification issues when $p$ or $q$ are allowed to be infinite. We shall call $d$ the memory parameter of $\left\{X_{t}\right\}$; with $d=0$ indicating short memory, $0<d<1 / 2$ long memory, and $-1 / 2<d<0$ antipersistence.

To conduct inference on $\mu$, define the sample mean $\bar{X}:=n^{-1} \sum_{j=1}^{n} X_{j}$ which satisfies

$$
\operatorname{var}\left(n^{1 / 2-d} \bar{X}\right)=n^{-1-2 d} \int_{-\pi}^{\pi}\left(\frac{\sin (n \lambda / 2)}{\sin (\lambda / 2)}\right)^{2} f(\lambda) \mathrm{d} \lambda .
$$

As $n \rightarrow \infty$, we can use assumption (1.1) and a change of variable of integration to get the convergence

$$
\operatorname{var}\left(n^{1 / 2-d} \bar{X}\right) \rightarrow s_{X}^{2}:=b_{0} \int_{-\infty}^{\infty}\left(\frac{\sin (\lambda / 2)}{\lambda / 2}\right)^{2}|\lambda|^{-2 d} \mathrm{~d} \lambda=b_{0} p(d),
$$

where we have the continuous function

$$
p(d):= \begin{cases}2 \frac{\Gamma(1-2 d) \sin (\pi d)}{d(1+2 d)}, & \text { if } d \neq 0, \\ 2 \pi, & \text { if } d=0 .\end{cases}
$$


We notice from (1.2) that $s_{X}^{2}$ is just a scaling of $b_{0}$ by the function $p(d)$, so in the usual short memory case of $d=0$ we get

$$
s_{X}^{2}=2 \pi f(0) \quad \text { and } \quad b_{0}=f(0) .
$$

In general, the problem of the estimation of the long-run variance $s_{X}^{2}$ is closely related to the estimation of $d$ and $b_{0} \equiv \lim _{\lambda \rightarrow 0}|\lambda|^{2 d} f(\lambda)$ appearing in (1.1). The $\mathrm{HAC}$ and MAC procedures mentioned at the start of this section hinge on the estimation of the long run variance $s_{X}^{2}$.

We will consider the behaviour of the estimators under two alternative sets of assumptions. The first one is stronger than the second one. It allows the derivation of asymptotic expansions and the resulting investigation of MSE-optimal bandwidth rates. The second one is sufficient to establish the consistency of the estimators for a wide class of stationary sequences. It allows the use of estimates of $s_{X}$ for robust SEs for $\bar{X}$. The second type of conditions are very weak, so they yield only consistency and are not sufficient to obtain other asymptotic results. 2 The first set of assumptions is common for HAC and MAC:

Assumption L. $\left\{X_{t}\right\}$ is a linear sequence

$$
X_{t}=\mu+\sum_{j=0}^{\infty} \psi_{j} \varepsilon_{t-j}, \quad t \in \mathbb{Z},
$$

where $\sum_{j=0}^{\infty} \psi_{j}^{2}<\infty, \mu$ is a real number and $\left\{\varepsilon_{j}\right\}$ are i.i.d. random variables with zero mean and unit variance. Moreover, the spectral density $f(\lambda)$ of $\left\{X_{t}\right\}$ has the property

$$
f(\lambda)=|\lambda|^{-2 d} g(\lambda),
$$

where $d \in(-1 / 2,1 / 2)$ and $g(\cdot)$ is a continuous bounded function such that $g(\lambda)=$ $b_{0}\left(1+O\left(|\lambda|^{2}\right)\right)$ as $\lambda \rightarrow 0$ and $b_{0}=g(0)>0$.

Let $\widehat{s}_{X}^{2}$ be a consistent estimator of $s_{X}^{2}$. Under condition (1.4), the t-ratio for the sample mean $\bar{X}$ satisfies

$$
\mathrm{t}:=n^{1 / 2-d} \frac{(\bar{X}-\mu)}{\widehat{s}_{X}} \stackrel{d}{\rightarrow} \mathrm{N}(0,1), \quad n \rightarrow \infty
$$

so that a consistent $\mathrm{HAC}$ or MAC estimator of $s_{X}^{2}$ allows inference on $\mu$.

For HAC, the second type of assumptions (to establish consistency) is:

Assumption M. $\left\{X_{t}\right\}$ is a fourth order stationary process such that, for some $d \in(-1 / 2,1 / 2)$ and $c \neq 0$,

$$
\gamma_{j} \sim c j^{2 d-1} \text { if } d \neq 0, \quad \sum_{j=-\infty}^{\infty}\left|\gamma_{j}\right|<\infty \text { if } d=0,
$$


where $\gamma_{j}:=\operatorname{cov}\left(X_{t}, X_{t+j}\right)$; and

$$
\sum_{h, r, s=-\infty}^{\infty}|\kappa(h, r, s)| \leq C \text { if } d<0, \quad \sup _{h} \sum_{r, s=-n}^{n}|\kappa(h, r, s)| \leq C n^{2 d} \text { if } d \geq 0,
$$

where $C$ denotes a generic constant and $\kappa(h, r, s)$ is a fourth-order cumulant defined by $\kappa(h, r, s):=\mathrm{E}\left(X_{k} X_{k+h} X_{k+r} X_{k+s}\right)-\left(\gamma_{h} \gamma_{r-s}+\gamma_{r} \gamma_{h-s}+\gamma_{s} \gamma_{h-r}\right)$. In addition, if $d<0$, then $f(\lambda) \leq C|\lambda|^{-2 d}, \lambda \in[-\pi, \pi]$.

For MAC, the second type of assumptions differs from Assumption M and is straightforward to discuss at the end of Section 3.

\section{Asymptotic properties of HAC-type estimators}

In this section, we first adapt the HAC estimator to allow for long memory and antipersistence, introducing two HAC-type estimators. Then, we analyze their properties under Assumption L that $\left\{X_{t}\right\}$ is a linear process, presenting limiting distributions and asymptotic expansions for the estimators. To the best of our knowledge, the asymptotic normality of the HAC estimator was investigated in the literature only in the short memory case of $d=0$ and under the assumption that $\mathrm{E}\left(\varepsilon_{t}^{4}\right)<\infty$. Our Theorem 2.1(a) will require for $\left\{\varepsilon_{t}\right\}$ the existence of only a moment of order $2+\delta$ (for some $\delta>0$ ), which is a new result in the field. It also shows that, under the strong persistence $1 / 4<d<1 / 2$, the asymptotic distribution will be non-Gaussian.

Finally, we show that Assumption M guarantees consistency (but not necessarily the other properties) of the estimators.

Let

$$
\widetilde{\gamma}_{j}:=n^{-1} \sum_{t=1}^{n-j}\left(X_{t}-\mathrm{E}\left(X_{t}\right)\right)\left(X_{t+j}-\mathrm{E}\left(X_{t}\right)\right), \quad 0 \leq j<n
$$

be the sample autocovariances of $\left\{X_{t}\right\}$ centered around $\mathrm{E}\left(X_{t}\right)$, and

$$
\bar{\gamma}_{j}:=n^{-1} \sum_{t=1}^{n-j}\left(X_{t}-\bar{X}\right)\left(X_{t+j}-\bar{X}\right), \quad 0 \leq j<n
$$

the sample autocovariances of $\left\{X_{t}\right\}$ centered around the sample mean $\bar{X}$.

Define

$$
\widetilde{s}_{q}^{2}(d):=q^{-1-2 d} \sum_{i, j=1}^{q} \widetilde{\gamma}_{|i-j|}=q^{-2 d}\left(\widetilde{\gamma}_{0}+2 \sum_{k=1}^{q}(1-k / q) \widetilde{\gamma}_{k}\right)
$$


which uses a known (or correctly hypothesized) $\mathrm{E}\left(X_{t}\right)$, and

$$
\bar{s}_{q}^{2}(d):=q^{-1-2 d} \sum_{i, j=1}^{q} \bar{\gamma}_{|i-j|}=q^{-2 d}\left(\bar{\gamma}_{0}+2 \sum_{k=1}^{q}(1-k / q) \bar{\gamma}_{k}\right)
$$

where the mean is estimated unrestrictedly, and assume that the bandwidth parameter $q$ satisfies

$$
q \rightarrow \infty, \quad q=O\left(n^{1-\epsilon}\right)
$$

for some $\epsilon>0$. The difference between the stochastic expansions of the two estimators will reveal just how much is the impact of estimating $\mathrm{E}\left(X_{t}\right)$. The asymptotically-optimal choice of $q$ will arise from the first theorem below. To make $\widetilde{s}_{q}^{2}(d)$ and $\bar{s}_{q}^{2}(d)$ operational, we can employ any estimator $\widehat{d}$ that is consistent at the rate of $\log n$ or faster, calculating $\widetilde{s}_{q}^{2}(\widehat{d})$ and $\bar{s}_{q}^{2}(\widehat{d})$. This is a very weak condition, and two such estimators of $\widehat{d}$ will be discussed later in Section 3 .

We start by making Assumption L. In addition, to establish the main theorem of this section, we need to assume that the coefficients $\psi_{j}$ decay as

$$
\begin{gathered}
\psi_{j}=c j^{-1+d}\left(1+O\left(j^{-1}\right)\right), c \neq 0, \text { if } d \neq 0 ; \quad \sum_{j=0}^{\infty} \psi_{j}=0 \text { if } d<0 ; \text { and } \\
\sum_{j=n}^{\infty}\left|\psi_{j}\right|=O\left(n^{-2}\right) \text { if } d=0 .
\end{gathered}
$$

Such additional requirements are satisfied, for example, by $X_{t} \sim \operatorname{ARIMA}(p, d, q)$ where $d \in(-1 / 2,1 / 2)$. We now derive asymptotic expansions for the estimators $\widetilde{s}_{q}^{2}(\widehat{d})$ and $\bar{s}_{q}^{2}(\widehat{d})$, where the bias will be expressed in terms of

$$
B:=\frac{1}{2} \int_{-\infty}^{\infty}\left(\frac{f(\lambda)}{\sin ^{2}(\lambda / 2)} 1_{|\lambda| \leq \pi}-\frac{b_{0}|\lambda|^{-2 d}}{(\lambda / 2)^{2}}\right) \mathrm{d} \lambda .
$$

In the case of $-1 / 2<d<1 / 4$, these HAC estimators have Gaussian limit distributions. However, if $1 / 4<d<1 / 2$, then the limit can be written in terms of a random variable given by the double Itô-Wiener integral

$$
R(d):=\int_{\mathbb{R}^{2}}^{\prime \prime} \frac{\mathrm{e}^{\mathrm{i}\left(x_{1}+x_{2}\right)}-1}{\mathrm{i}\left(x_{1}+x_{2}\right)}\left|x_{1}\right|^{-d}\left|x_{2}\right|^{-d} Z\left(\mathrm{~d} x_{1}\right) Z\left(\mathrm{~d} x_{2}\right),
$$

where $Z(\mathrm{~d} x)$ is a standard Gaussian complex measure $(Z(-\mathrm{d} x)$ is the conjugate of $Z(\mathrm{~d} x))$ with mean zero and variance $\mathrm{E}\left(|Z(\mathrm{~d} x)|^{2}\right)=\mathrm{d} x$. The limit variable $R(d)$ has a (non-Gaussian) Rosenblatt distribution and is well-defined when $1 / 4<d<1 / 2$. The symbol $\int_{\mathbb{R}^{2}}^{\prime \prime}$ indicates that one does not integrate on the diagonals $x_{1}= \pm x_{2}$. 
Theorem 2.1. Supppose that $\left\{X_{t}\right\}$ satisfies Assumption L and (2.4)-(2.5), and that $\widehat{d}$ is an estimator of $d$ such that

$$
(\widehat{d}-d) \log n=o_{p}(1)
$$

(a) If $-1 / 2<d<1 / 4$ and $\mathrm{E}\left(\left|\varepsilon_{t}\right|^{2+\delta}\right)<\infty$, for some $\delta>0$, then, as $n \rightarrow \infty$,

$$
\widetilde{s}_{q}^{2}(\widehat{d})-s_{X}^{2}=(q / n)^{1 / 2} Z_{n}+q^{-1-2 d} B+o_{p}\left((q / n)^{1 / 2}\right)+o_{p}\left(q^{-1-2 d}\right)
$$

and

$$
\bar{s}_{q}^{2}(\widehat{d})-s_{X}^{2}=(q / n)^{1 / 2} Z_{n}+q^{-1-2 d} B+o_{p}\left((q / n)^{1 / 2}\right)+o_{p}\left(q^{-1-2 d}\right),
$$

where $Z_{n} \stackrel{d}{\rightarrow} \mathrm{N}\left(0, v_{d}^{2}\right)$,

$$
v_{d}^{2}:=8 \pi b_{0}^{2} \int_{0}^{\infty}\left(\frac{\sin (x / 2)}{x / 2}\right)^{4} x^{-4 d} \mathrm{~d} x= \begin{cases}16 \pi b_{0}^{2} \frac{2\left(2^{1+4 d}-1\right) \pi}{\Gamma(4+4 d) \cos (2 \pi d)}, & \text { if } d \neq 0 \\ 16 \pi^{2} b_{0}^{2} / 3=4 s_{X}^{4} / 3, & \text { if } d=0\end{cases}
$$

and it is understood that $\lim _{d \rightarrow-1 / 4}\left(2^{1+4 d}-1\right) \pi / \cos (2 \pi d)=\log 4$.

(b) If $1 / 4<d<1 / 2, \mathrm{E}\left(\varepsilon_{t}^{4}\right)<\infty$ and $g(\lambda)$ in (1.4) has bounded derivative, then

$$
\widetilde{s}_{q}^{2}(\widehat{d})-s_{X}^{2}=(q / n)^{1-2 d} \widetilde{Z}_{n}+q^{-1-2 d} B+o_{p}\left((q / n)^{1-2 d}\right)+o_{p}\left(q^{-1-2 d}\right),
$$

where

$$
\widetilde{Z}_{n}:=n^{-2 d} \sum_{j=1}^{n}\left(X_{j}^{2}-\mathrm{E}\left(X_{j}^{2}\right)\right) \stackrel{d}{\rightarrow} 2 \pi b_{0} R(d)
$$

whereas $\bar{s}_{q}^{2}(\widehat{d})$ has the property

$$
\bar{s}_{q}^{2}(\widehat{d})-\widetilde{s}_{q}^{2}(\widehat{d})=O_{p}\left((q / n)^{1-2 d}\right) .
$$

Under the additional assumption that $\mathrm{E}\left(\varepsilon_{t}^{4}\right)<\infty$, the MSEs of HAC-type estimators exist and are minimized asymptotically by

$$
q \propto \begin{cases}n^{1 /(3+4 d)}, & -1 / 2<d<1 / 4 \\ n^{1 / 2-d}, & 1 / 4<d<1 / 2\end{cases}
$$

where $\propto$ denotes proportionality. We now list other comments and implications arising from Theorem 2.1:

REMARK 2.1. Since $\mathrm{E}\left(Z_{n}\right)=\mathrm{E}\left(\widetilde{Z}_{n}\right)=0$, the asymptotic bias of the estimators is given by $q^{-1-2 d} B$. It tends to zero as $n$ (hence $q$ ) tends to infinity. 
REMARK 2.2. When $-1 / 2<d<1 / 4$, the convergence $\bar{s}_{q}^{2}(\widehat{d}) \stackrel{d}{\longrightarrow} s_{X}^{2}=p(d) b_{0}$ implies that $v_{d}^{2}$ can be consistently estimated by replacing $b_{0}$ by $\bar{s}_{q}^{2}(\widehat{d}) / p(\widehat{d})$ in $(2.11)$.

REMARK 2.3. If $d<1 / 4$, then estimates with known and estimated mean have the same asymptotic properties. However, if $d>1 / 4$, then the rate of convergence of the sample mean to $\mu$ is rather slow, and replacing $\mu$ by $\bar{X}$ leads to an additional term in the limiting distribution of the HAC estimator whose consistency is nevertheless unaffected. In the context of hypothesis testing about the mean $\mu$, one can estimate the long run variance by treating $\mu$ as unknown and estimating it by the sample mean. Alternatively, one can compute the long-run variance under the null hypothesis, treating $\mu$ as known. This will improve the size but may have an adverse effect on the finite-sample power of tests based on HAC estimators.

REMARK 2.4. As a general rule, convergence in distribution does not necessarily imply a corresponding convergence for moments such as the MSE. However, our proofs are based on $L_{2}$ expansions for which this implication holds if we make the additional assumption that $\mathrm{E}\left(\varepsilon_{t}^{4}\right)<\infty$, hence our stated results for the asymptotic bias and variance. Note that for the validity of asymptotic expansions (2.9)-(2.10), only $2+\delta$ moments of $\left\{\varepsilon_{t}\right\}$ are needed.

REMARK 2.5. If $\left\{X_{t}\right\}$ is a nonlinear process, then Theorem 2.1 might not hold. For example, the nonlinear transformation $X_{t}=\mathrm{e}^{\xi_{t}}$ of a linear process $\left\{\xi_{t}\right\}$ will, in general, increase the bias of estimators. Therefore, the optimal $q$ minimizing the MSE might also change in this case.

Relaxing Assumption L, we obtain the following concistency result.

Theorem 2.2. Suppose that $q \rightarrow \infty, q=O\left(n^{1 / 2}\right)$, that Assumption $M$ holds, and that $\widehat{d}-d=o_{p}(1 / \log n)$. Then,

$$
\bar{s}_{q}^{2}(\widehat{d}) \stackrel{p}{\rightarrow} s_{X}^{2}, \quad \widetilde{s}_{q}^{2}(\widehat{d}) \stackrel{p}{\longrightarrow} s_{X}^{2}, \quad \text { as } n \rightarrow \infty
$$

\section{Robinson's MAC estimator}

In this section, we derive the asymptotic properties of Robinson's MAC estimator of $s_{X}^{2}=p(d) b_{0}$, where $p(d)$ is given by (1.3). We shall show that the asymptotic properties of the MAC estimator do not depend on the memory parameter $d$, and its asymptotic distribution is always Gaussian. Hence, it is more robust than HAC to 
the bandwidth selection in practice, something that will be illustrated numerically in the subsequent section. Define

$$
\widehat{s}_{m}^{2}(d):=p(d) \widehat{b}_{m}(d)
$$

where

$$
\widehat{b}_{m}(d):=m^{-1} \sum_{j=1}^{m} \lambda_{j}^{2 d} I_{n}\left(\lambda_{j}\right)
$$

is a consistent estimator of $b_{0}$,

$$
I_{n}\left(\lambda_{j}\right):=(2 \pi n)^{-1}\left|\sum_{t=1}^{n} \mathrm{e}^{\mathrm{i} t \lambda_{j}} X_{t}\right|^{2}
$$

is the periodogram, $\lambda_{j}=2 \pi j / n$ are the Fourier frequencies, and the bandwidth parameter $m$ satisfies $m \rightarrow \infty$ and $m=o\left(n /(\log n)^{2}\right)$.

This estimator has a number of features. First, it does not require estimation of the unknown mean $\mathrm{E}\left(X_{t}\right)$ since the periodogram is self-centring at the Fourier frequencies $\lambda_{j}$. Contrast this with HAC estimators; see also Remark 2.3. Second, as the following theorem will show, the bias and asymptotic distribution of the estimator do not depend on $d \in(-1 / 2,1 / 2)$, and the asymptotic distribution is always Gaussian.

In addition to Assumption L, we will need the condition that $\alpha(\lambda):=$ $\sum_{j=0}^{\infty} \mathrm{e}^{\mathrm{i} j \lambda} \psi_{j}$ satisfies

$$
\frac{\mathrm{d}}{\mathrm{d} \lambda} \alpha(\lambda)=O(|\alpha(\lambda)| / \lambda), \quad \text { as } \quad \lambda \rightarrow 0^{+},
$$

in order to derive the CLT in the following theorem.

Theorem 3.1. Suppose that $\left\{X_{t}\right\}$ satisfies Assumption L with $\mathrm{E}\left(\varepsilon_{t}^{4}\right)<\infty$ and (3.1). Assume that $\widehat{d}$ is an estimator of $d$ such that $\widehat{d}-d=o_{p}(1 / \log n)$. Then

$$
\begin{aligned}
\widehat{s}_{m}^{2}(\widehat{d})-s_{X}^{2}= & m^{-1 / 2} s_{X}^{2} T_{n}+2(\widehat{d}-d)\left(\log \lambda_{m}\right) s_{X}^{2}\left(1+o_{p}(1)\right) \\
& +O_{p}\left((m / n)^{2}\right)+o_{p}\left(m^{-1 / 2}\right)
\end{aligned}
$$

where

$$
T_{n} \stackrel{d}{\rightarrow} \mathrm{N}(0,1)
$$

The parameter $d$ can be estimated, for example, by the local Whittle estimator

$$
\widehat{d}:=\operatorname{argmin}_{d \in[-1 / 2,1 / 2]} U_{n}(d),
$$


which minimizes the objective function

$$
U_{n}(d):=\log \left(\frac{1}{m_{w}} \sum_{j=1}^{m_{w}} j^{2 d} I_{n}\left(\lambda_{j}\right)\right)-\frac{2 d}{m_{w}} \sum_{j=1}^{m_{w}} \log j,
$$

with bandwidth parameter $m_{w}$ such that $m_{w} \rightarrow \infty$ and $m_{w}=o\left(n /(\log n)^{2}\right)$. We use the notation $m_{w}$ for the bandwidth of the local Whittle estimator, stressing that it can be set to values that can differ from the bandwidth $m$ used in $\widehat{s}_{m}^{2}$. If $m_{w}=o\left(n^{4 / 5}\right)$, then Robinson (1995b) showed that under the assumptions of Theorem 3.1

$$
\sqrt{m_{w}}(\widehat{d}-d) \stackrel{d}{\rightarrow} \mathrm{N}(0,1 / 4) .
$$

For the estimation of $d$, the log-periodogram estimator can be used as an alternative to the local Whittle estimator; see Robinson (1995a).

We now turn to the MSE of $\widehat{s}_{m}^{2}(\widehat{d})$, when $\widehat{d}$ is the local Whittle estimator. Let $m_{w}=O\left(n^{4 / 5}\right)$ and $m=O\left(n^{4 / 5}\right)$, since we only need a consistency rate rather than a CLT to analyze the decline of the MSE as $n$ increases. Under Assumption L, $\mathrm{E}\left((\widehat{d}-d)^{2}\right)=O\left(m_{w}^{-1}\right)$. Therefore,

$$
\left(\widehat{s}_{m}^{2}(\widehat{d})-s_{X}^{2}\right)^{2}=O_{p}\left(\frac{1}{m}+\frac{(\log n)^{2}}{m_{w}}\right)
$$

by (3.2). Since (3.5) is derived using an $L_{2}$ approximation, a more detailed analysis shows that the MSE is $O\left(m^{-1}+(\log n)^{2} m_{w}^{-1}\right)$, hence decreasing in $m$. The MSEoptimal bandwidth is therefore the one taking $m$ that grows at the maximal rate of $n^{4 / 5}$.

In general, without recourse to Assumption L, the consistency of Robinson's MAC estimator follows immediately from $\widehat{d} \stackrel{p}{\rightarrow} d$ and $\widehat{b}_{m}(\widehat{d}) \stackrel{p}{\rightarrow} b_{0}$. The estimators $\widehat{d}$ and $\widehat{b}_{m}(\widehat{d})$ are consistent under very weak general assumptions, which do not assume Gaussianity or linearity of $\left\{X_{t}\right\}$; see Dalla et al. (2006) and Abadir et al. (2007). For example, if $d=0$ and (1.4) holds, then such consistency follows under the assumption $\sum_{h, r, s=-\infty}^{\infty}|\kappa(h, r, s)| \leq C$; see Corollary 1 of Dalla et al. (2006).

\section{Simulation results}

The objective of this section is to illustrate the asymptotic results for the HAC and MAC estimators $\bar{s}_{q}^{2}(\widehat{d})$ and $\widehat{s}_{m}^{2}(\widehat{d})$, to examine their finite-sample performance, and to give advice on how to choose the bandwidth parameters in practical applications. 
We focus on the MSE because the primary use of these estimators is the consistent estimation of the long-run variance $s_{X}^{2}$ used in various statistics; e.g. in the denominator $\widehat{s}_{X}$ of HAC and MAC robust t-ratios

$$
\mathrm{t}:=n^{1 / 2-d} \frac{(\bar{X}-\mu)}{\widehat{s}_{X}} \stackrel{d}{\rightarrow} \mathrm{N}(0,1), \quad n \rightarrow \infty .
$$

For this reason, we also consider the closeness of HAC and MAC robust t-ratios to their limiting normal distributions; see Velasco and Robinson (2001) for expansions relating to t-ratios using smoothed autocovariance estimates for $f(0)$. We study the coverage probabilities (CPs) of $95 \%$ asymptotic confidence intervals (CIs) for $\mu$, considering how the choice of bandwidths affects the closeness of CPs to the nominal 95\% level based on the limiting normal distribution of the t-ratio.

We let $\left\{X_{t}\right\}$ be a linear Gaussian $\operatorname{ARIMA}(1, d, 0)$ process with unit standard deviation, for different values of $\rho$ (AR parameter) and $d$. We link $d$ to $s_{X}^{2}$, the object of our analysis, by means of (1.2)-(1.3). Throughout the simulation exercise, the number of replications is 5,000. We consider three sample sizes $n=250,500,1000$ and we estimate the parameter $d$ using the local Whittle estimator $\hat{d}$ with bandwidth $m_{w}=\left\lfloor n^{0.65}\right\rfloor$. We do not report the results for $m_{w}=\left\lfloor n^{0.5}\right\rfloor,\left\lfloor n^{0.8}\right\rfloor$ because they are dominated by $m_{w}=\left\lfloor n^{0.65}\right\rfloor$.

Table 1 contains the MSE of the HAC estimator $\bar{s}_{q}^{2}(\widehat{d})$ calculated for different values of the bandwidth $q$. The minimum-MSE value for each $d$ and $n$ is highlighted by shaded gray boxes. The results for these optima are so scattered across the table, that in practice it will be difficult to achieve them.

Table 2 reports the MSEs of the HAC estimator $\bar{s}_{q}^{2}(\widehat{d})$ when $q$ is chosen according to the asymptotically-optimal rule (2.14). It gives MSEs comparable to the optimal MSEs of Table 1, except when $d$ and $\rho$ are simultaneously large. In this case, the cost in terms of the MSE can be substantial.

Table 3 contains the MSE of the MAC estimator $\widehat{s}_{m}^{2}(\widehat{d})$ calculated for different values of the bandwidth $m$. It reveals the accuracy of the simple bandwidth rule that resulted from (3.5): almost all the optima are for $m=O\left(n^{4 / 5}\right)$ and, in the four exceptions (shaded boxes), there is little loss in nevertheless sticking to $m=O\left(n^{4 / 5}\right)$. Both Tables 2 and 3 show that the MSEs of HAC and MAC estimators usually increase when $|d|$ or $|\rho|$ increase.

Tables 4 and 5 report CPs for $\mu$ using, respectively, the HAC estimator $\bar{s}_{q}^{2}(\widehat{d})$ with $q$ chosen by the rule (2.14) and the MAC estimator with various bandwidths $m$. HAC and MAC estimators gives comparable CPs, which are slightly better 
for MAC. CPs approach the nominal $95 \%$ level as sample size increases. They are close to the $95 \%$ level except when $d \rightarrow 0.5$ or when $\rho$ becomes negative. The bandwidth $m=\left\lfloor n^{0.8}\right\rfloor$ tends to give better CPs for MAC, and this is in line with the recommendations of Table 3 .

Because of the specificity of MC studies to the generating process that is used, it is recommended in practice that the user tries also bandwidths that are smaller than the maximum allowable $m=\left\lfloor n^{0.8}\right\rfloor$ which we recommended. This could be used to check the stability of the estimator as $m$ varies near its (unknown) optimal value. For example, data that are not generated by a linear process (such as ARIMA) require smaller bandwidths like $\left\lfloor n^{0.7}\right\rfloor$; see Dalla et al. (2006).

\section{Concluding Remarks}

In this paper, the properties of two alternative types of estimators of the long-run variance have been derived. The first one is an extension of the widely used Bartlettkernel HAC estimator, while the second one is the frequency-based MAC estimator suggested by Robinson (2005). We give guidance on how to choose the bandwidths in practice, for each estimator. The calculation of both estimators is numerically straightforward, and allows for the possibility of long-memory or antipersistence in the data.

Our theoretical results explain that the HAC estimator is sensitive to the selection of the bandwidth $q$, since the order of $q$ minimizing the MSE depends on the extent of the memory in the series. This problem often complicates bandwidth selection in applied work. The MAC estimator is more robust to the choice of the bandwidth, which does not depend on the memory. The simulation study confirms this analytical finding.

On the other hand, the paper does not provide a theory of deriving optimal estimators, e.g. under MSE-optimality or closeness to normality of the Studentized t-ratio for $\mu$. We have studied two types of estimators without establishing whether or not they are dominated by others, but the asymptotic normality of the MAC estimator for $d \in(-1 / 2,1 / 2)$ is an encouraging sign, and so is the good simulation performance of the two estimators. 


\section{Appendix}

\section{A Proofs of the theorems, auxiliary lemmas and propo- sitions}

There are four subsections. The first proves the results relating to the theorems of Section 2, while the second proves the theorem of Section 3. For the first theorem, we need lemmas that are derived in the third subsection, and propositions that are obtained in the fourth one. We require these auxiliary results here, but they can also be of use beyond our paper.

Throughout this section, we take $a_{n} \sim b_{n}$ to mean that $a_{n} / b_{n} \rightarrow 1$ as $n \rightarrow \infty$.

\section{A.1 Proof of Theorems 2.1 and 2.2}

Proof of Theorem 2.1. By definitions (2.1), (2.2), and (2.8)

$$
\widetilde{s}_{q}^{2}(\widehat{d})=\widetilde{s}_{q}^{2}(d)\left(1+o_{p}(1)\right) \quad \text { and } \quad \bar{s}_{q}^{2}(\widehat{d})=\bar{s}_{q}^{2}(d)\left(1+o_{p}(1)\right)
$$

Condition (2.8) and asymptotic results derived for $\bar{s}_{q}^{2}(d)$ and $\bar{s}_{q}^{2}(d)$ below then allows us to replace $\widetilde{s}_{q}^{2}(\widehat{d})$ and $\bar{s}_{q}^{2}(\widehat{d})$ by $\widetilde{s}_{q}^{2}(d)$ and $\bar{s}_{q}^{2}(d)$ in the statement of the theorem without altering the expansions, so we will prove the theorem for $\widetilde{s}_{q}^{2}(d)$ and $\bar{s}_{q}^{2}(d)$. Also, observe that

$$
\widetilde{\gamma}_{j}=\int_{-\pi}^{\pi} \mathrm{e}^{\mathrm{i} j \lambda} I_{n}(\lambda) \mathrm{d} \lambda, \quad \bar{\gamma}_{j}=\int_{-\pi}^{\pi} \mathrm{e}^{\mathrm{i} j \lambda} \bar{I}_{n}(\lambda) \mathrm{d} \lambda, \quad 0 \leq j<n
$$

where

$$
I_{n}(\lambda):=(2 \pi n)^{-1}\left|\sum_{t=1}^{n} \mathrm{e}^{\mathrm{i} t \lambda}\left(X_{t}-\mathrm{E}\left(X_{t}\right)\right)\right|^{2}, \quad \bar{I}_{n}(\lambda):=(2 \pi n)^{-1}\left|\sum_{t=1}^{n} \mathrm{e}^{\mathrm{i} t \lambda}\left(X_{t}-\bar{X}\right)\right|^{2}
$$

are the corresponding periodograms. Therefore,

$$
\widetilde{s}_{q}^{2}(d)=\int_{-\pi}^{\pi} b_{n}(\lambda) I_{n}(\lambda) \mathrm{d} \lambda
$$

and

$$
\bar{s}_{q}^{2}(d)=\int_{-\pi}^{\pi} b_{n}(\lambda) \bar{I}_{n}(\lambda) \mathrm{d} \lambda,
$$

where

$$
b_{n}(\lambda):=q^{-1-2 d}\left|\sum_{t=1}^{q} \mathrm{e}^{\mathrm{i} t \lambda}\right|^{2}=q^{-1-2 d}\left(\frac{\sin (q \lambda / 2)}{\sin (\lambda / 2)}\right)^{2}
$$


is the renormalized Fejér kernel.

By (A.1) and (A.2), we can write $\bar{s}_{q}^{2}(d)=\widetilde{s}_{q}^{2}(d)+R_{n}$, where

$$
R_{n}:=\int_{-\pi}^{\pi} b_{n}(\lambda)\left(\bar{I}_{n}(\lambda)-I_{n}(\lambda)\right) \mathrm{d} \lambda .
$$

In Lemma A.4, we will show that $\mathrm{E}\left(\left|R_{n}\right|\right) \leq C\left((q / n)^{1-2 d}+(q / n)\right)$. Hence,

$$
\bar{s}_{q}^{2}(d)=\widetilde{s}_{q}^{2}(d)+O_{p}\left((q / n)^{1-2 d}\right) .
$$

If $-1 / 2<d<1 / 4$, then $(q / n)^{1-2 d}=o\left((q / n)^{1 / 2}\right)$, and we can write (A.5) as

$$
\bar{s}_{q}^{2}(d)-s_{X}^{2}=(q / n)^{1 / 2} Z_{n}+\left(\int_{-\pi}^{\pi} b_{n}(\lambda) f(\lambda) \mathrm{d} \lambda-s_{X}^{2}\right)+o_{p}\left((q / n)^{1 / 2}\right),
$$

where

$$
Z_{n}:=(n / q)^{1 / 2}\left(\widetilde{s}_{q}^{2}(d)-\int_{-\pi}^{\pi} b_{n}(\lambda) f(\lambda) \mathrm{d} \lambda\right) .
$$

By Proposition A.1, $Z_{n} \stackrel{d}{\longrightarrow} \mathrm{N}\left(0, v_{d}^{2}\right)$, where $v_{d}^{2}$ is given by (2.11), whereas by Proposition A.2,

$$
\int_{-\pi}^{\pi} b_{n}(\lambda) f(\lambda) \mathrm{d} \lambda=s_{X}^{2}+q^{-1-2 d} B+o\left(q^{-1-2 d}\right),
$$

which proves (2.9) and (2.10).

In the case $1 / 4<d<1 / 2$, write

$$
\widetilde{s}_{q}^{2}(d)-s_{X}^{2}=\left(\widetilde{s}_{q}^{2}(d)-\mathrm{E}\left(\widetilde{s}_{q}^{2}(d)\right)\right)+\mathrm{E}\left(\widetilde{s}_{q}^{2}(d)\right)-s_{X}^{2} .
$$

Proposition A.3 derives the asymptotic bias $\mathrm{E}\left(\widetilde{s}_{q}^{2}(d)\right)-s_{X}^{2}=q^{-1-2 d} B+o\left(q^{-1-2 d}\right)+$ $o\left((q / n)^{1-2 d}\right)$ and shows that the stochastic term exhibits the nonstandard asymptotic behavior

$$
(n / q)^{1-2 d}\left(\widetilde{s}_{q}^{2}(d)-\mathrm{E}\left(\widetilde{s}_{q}^{2}(d)\right)\right)=n^{-2 d} \sum_{j=1}^{n}\left(X_{j}^{2}-\mathrm{E}\left(X_{j}^{2}\right)\right)+o_{p}(1) \stackrel{d}{\longrightarrow} 2 \pi b_{0} R(d) .
$$

Thus, the term on the left-hand side above can be approximated by the normalized sum $n^{-2 d} \sum_{j=1}^{n}\left(X_{j}^{2}-\mathrm{E}\left(X_{j}^{2}\right)\right)$ of strongly dependent variables $X_{t}^{2}$ which has a nonGaussian limit distribution. These relations imply (2.12) and (2.13).

Proof of Theorem 2.2. The condition $\widehat{d}-d=o_{p}(1 / \log n)$ allows us to prove the theorem for $\widetilde{s}_{q}^{2}(d)$ and $\bar{s}_{q}^{2}(d)$ instead of $\widetilde{s}_{q}^{2}(\widehat{d})$ and $\bar{s}_{q}^{2}(\widehat{d})$. For $d \geq 0$, convergence (2.15) was shown in Giraitis et al. (2003, Theorem 3.1). For $d<0$,

$$
q^{2 d} \bar{s}_{q}^{2}=\sum_{|j|<q}\left(1-\frac{|j|}{q}\right) \widetilde{\gamma}_{j}+\sum_{|j|<q}\left(1-\frac{|j|}{q}\right) \delta_{j}=: v_{n, 1}+v_{n, 2},
$$


where

$$
\begin{aligned}
& \widetilde{\gamma}_{j}=n^{-1} \sum_{i=1}^{n-|j|}\left(X_{i}-\mu\right)\left(X_{i+|j|}-\mu\right), \\
& \delta_{j}=\left(1-\frac{|j|}{n}\right)\left(\bar{X}_{n}-\mu\right)^{2}-n^{-1}\left(\bar{X}_{n}-\mu\right)\left(Z_{1, n-|j|}+Z_{|j|+1, n}\right),
\end{aligned}
$$

$\mu=\mathrm{E}\left(X_{t}\right)$, and $Z_{k, l}:=\sum_{i=k}^{l}\left(X_{i}-\mu\right)$. It suffices to show that

$$
q^{-2 d} v_{n, 1} \stackrel{p}{\rightarrow} s_{X}^{2} \quad \text { and } \quad q^{-2 d} v_{n, 2} \stackrel{p}{\longrightarrow} 0 .
$$

The verification of the relations $q^{-2 d} v_{n, 2} \stackrel{p}{\rightarrow} 0$ and $q^{-2 d} \mathrm{E}\left(v_{n, 1}\right) \rightarrow s_{X}^{2}$ is the same as in Giraitis et al. (2003).

To prove the convergence (A.6), it remains to check that $\mathrm{E}\left(\left(v_{n, 1}-\mathrm{E}\left(v_{n, 1}\right)\right)^{2}\right)=$ $o\left(q^{4 d}\right)$. We have $\mathrm{E}\left(\left(v_{n, 1}-\mathrm{E}\left(v_{n, 1}\right)\right)^{2}\right) \leq\left|R_{n}\right|+R_{n}^{\prime}$, where

$$
\begin{gathered}
R_{n}:=\sum_{|j|,\left|j^{\prime}\right|<q}\left(1-\frac{|j|}{q}\right)\left(1-\frac{\left|j^{\prime}\right|}{q}\right) n^{-2} \sum_{i=1}^{n-|j|} \sum_{i^{\prime}=1}^{n-\left|j^{\prime}\right|} d\left(i, i^{\prime}, j, j^{\prime}\right), \\
d\left(i, i^{\prime}, j, j^{\prime}\right):=\gamma_{i-i^{\prime}} \gamma_{i-i^{\prime}+|j|-\left|j^{\prime}\right|}+\gamma_{i-i^{\prime}-\left|j^{\prime}\right|} \gamma_{i-i^{\prime}+|j|}
\end{gathered}
$$

and

$$
\begin{aligned}
& R_{n}^{\prime}:=n^{-2} \sum_{|j|,\left|j^{\prime}\right| \leq q} \sum_{i=1}^{n-|j|} \sum_{i^{\prime}=1}^{n-\left|j^{\prime}\right|}\left|\kappa\left(|j|, i^{\prime}-i, i^{\prime}-i+\left|j^{\prime}\right|\right)\right| \\
& \leq n^{-2} \sum_{|j| \leq q} \sum_{i=1}^{n} \sum_{i^{\prime}, j^{\prime}=-\infty}^{\infty}\left|\kappa\left(|j|, i^{\prime}, j^{\prime}\right)\right| \leq C n^{-1}=o\left(q^{4 d}\right),
\end{aligned}
$$

by the assumption $\sum_{h, r, s=-\infty}^{\infty}|\kappa(h, r, s)|<\infty$ for $d<0,-1 / 2<d<0$ and $q=O(n)$.

To work out $R_{n}$, write $R_{n}=Q_{n}+Q_{n}^{\prime}$ where

$$
Q_{n}:=\sum_{|j|,\left|j^{\prime}\right|<q}\left(1-\frac{|j|}{q}\right)\left(1-\frac{\left|j^{\prime}\right|}{q}\right) n^{-2} \sum_{i=-\infty}^{\infty} \sum_{i^{\prime}=1}^{n-\left|j^{\prime}\right|} d\left(i, i^{\prime}, j, j^{\prime}\right),
$$

whereas $Q_{n}^{\prime}$ can be bounded by

$$
\left|Q_{n}^{\prime}\right| \leq C n^{-2} \sum_{|j|,\left|j^{\prime}\right|<q} \sum_{i>n-|j| \text { or }} \sum_{i \leq 0}^{n}\left|d\left(i, i^{\prime}, j, j^{\prime}\right)\right| .
$$

We split summation over $i$ into three regions: $n-|j| \leq i \leq n, i>n$, and $i \leq 0$. In the case of $n-|j| \leq i \leq n$, the order of this part of the sum is straightforward. 
Since $\left|\gamma_{j}\right| \leq C j^{-1+2 d}=: C_{j}$ for all $j \geq 1$, then for all $|j| \leq q, i>n$, and $1 \leq i^{\prime} \leq n$, we can bound

$$
\left|\gamma_{i-i^{\prime}}\right| \leq C_{n-i^{\prime}} \text { and }\left|\gamma_{i-i^{\prime}+|j|}\right| \leq C_{n-i^{\prime}},
$$

and, for all $|j| \leq q, i \leq 0$, and $1 \leq i^{\prime} \leq n$, we bound

$$
\left|\gamma_{i-i^{\prime}}\right| \leq C_{i^{\prime}} \text { and }\left|\gamma_{i-i^{\prime}-|j|}\right| \leq C_{i^{\prime}+1} .
$$

Since $\sum\left|\gamma_{j}\right|<\infty$ and $\sum C_{j}<\infty$, then

$$
\begin{aligned}
\left|Q_{n}^{\prime}\right| \leq C n^{-2}\left(\sum_{|j|<q} \sum_{i=n-|j|}^{n} \sum_{j^{\prime}=-\infty}^{\infty}\left|\gamma_{j^{\prime}}\right| \sum_{i^{\prime}=-\infty}^{\infty}\left|\gamma_{i^{\prime}}\right|+\sum_{|j|,\left|j^{\prime}\right|<q} \sum_{i^{\prime}=1}^{\infty} C_{i^{\prime}} \sum_{i=-\infty}^{\infty}\left|\gamma_{i}\right|\right) \\
\leq C q^{2} n^{-2}=o\left(q^{4 d}\right),
\end{aligned}
$$

because $-1 / 2<d<0$ and $q=O(n)$.

To estimate $Q_{n}$, denote $q_{k}:=\sum_{i=-\infty}^{\infty} \gamma_{i} \gamma_{k+i}=2 \pi \int_{-\pi}^{\pi} \mathrm{e}^{\mathrm{i} k x} f^{2}(x) \mathrm{d} x$. Then

$$
Q_{n}^{\prime}=n^{-1} \sum_{|j|,\left|j^{\prime}\right|<q}\left(1-\frac{|j|}{q}\right)\left(1-\frac{\left|j^{\prime}\right|}{q}\right)\left(q_{|j|-\left|j^{\prime}\right|}+q_{|j|+\left|j^{\prime}\right|}\right) .
$$

It remains to show that

$$
Q_{n}^{\prime}=o\left(q^{4 d}\right)
$$

Note that

$$
\begin{aligned}
& \left|R_{n}^{\prime}\right| \\
\leq & n^{-1}\left|\int_{-\pi}^{\pi} \sum_{|j|,\left|j^{\prime}\right|<q}\left(1-\frac{|j|}{q}\right)\left(1-\frac{\left|j^{\prime}\right|}{q}\right)\left(\mathrm{e}^{\mathrm{i}\left(|j|-\left|j^{\prime}\right|\right) x}+\mathrm{e}^{\mathrm{i}\left(|j|+\left|j^{\prime}\right|\right) x}\right) f^{2}(x) \mathrm{d} x\right| \\
\leq & C n^{-1} \int_{-\pi}^{\pi}\left(\left|\sum_{j=1}^{q} \mathrm{e}^{\mathrm{i} j x}\left(1-\frac{j}{q}\right)\right|^{2}+1\right) f^{2}(x) \mathrm{d} x .
\end{aligned}
$$

Summation by parts yields $\sum_{j=1}^{q} \mathrm{e}^{\mathrm{i} j x}(1-j / q)=q^{-1} \sum_{j=1}^{q-1} \sum_{l=1}^{j} \mathrm{e}^{\mathrm{i} l x}$, where

$$
\left|\sum_{l=1}^{j} \mathrm{e}^{\mathrm{i} l x}\right|=\left|\frac{\sin (j x / 2)}{\sin (x / 2)}\right| \leq C \frac{|j|}{1+j|x|} \leq \frac{C}{|x|}
$$

for all $j \geq 1$ and $|x| \leq \pi$. Set $\alpha=\max (4 d,-1+\epsilon)$ where $\epsilon>0$ will be chosen sufficiently small. Then $f^{2}(x) \leq C|x|^{-4 d} \leq C|x|^{-\alpha}$ and

$$
\begin{aligned}
\left|Q_{n}^{\prime}\right| & \leq C n^{-1}\left(q^{-1} \sum_{j=1}^{q-1} \int_{-\pi}^{\pi} j^{2}(1+j|x|)^{-2}|x|^{-\alpha} \mathrm{d} x+1\right) \\
& \leq C n^{-1}\left(q^{-1} \sum_{j=1}^{q-1}|j|^{1+\alpha} \int_{-\infty}^{\infty}(1+|x|)^{-2}|x|^{-\alpha} \mathrm{d} x+1\right) \leq C\left((q / n) q^{\alpha}+n^{-1}\right)=o\left(q^{4 d}\right)
\end{aligned}
$$

for $-1 / 2<d<0$, when $q=O(n)$ and $\epsilon>0$ is sufficiently small. 


\section{A.2 Proof of Theorem 3.1}

We show first that

$$
\widehat{b}_{m}(d)-b_{0}=m^{-1 / 2} b_{0} T_{n}+O_{p}\left((m / n)^{2} m^{-1 / 2}\right)+o_{p}\left(m^{-1 / 2}\right),
$$

where

$$
T_{n} \stackrel{d}{\rightarrow} \mathrm{N}(0,1)
$$

Write

$$
\widehat{b}_{m}=b_{0} m^{-1} \sum_{j=1}^{m} b_{0}^{-1} \lambda_{j}^{2 d} I_{n}\left(\lambda_{j}\right)=b_{0}\left(S_{n, 1}+S_{n, 2}\right),
$$

where

$$
S_{n, 1}:=m^{-1} \sum_{j=1}^{m}\left(b_{0}^{-1} \lambda_{j}^{2 d} I_{n}\left(\lambda_{j}\right)-2 \pi I_{\varepsilon, n}\left(\lambda_{j}\right)\right), \quad S_{n, 2}:=m^{-1} \sum_{j=1}^{m} 2 \pi I_{\varepsilon, n}\left(\lambda_{j}\right)
$$

and $I_{\varepsilon, n}\left(\lambda_{j}\right):=(2 \pi n)^{-1} \sum_{k=1}^{n} \mathrm{e}^{\mathrm{i} k \lambda_{j}} \varepsilon_{k}$.

Under the assumptions of the theorem, (4.8) of Robinson (1995b) implies that

$$
S_{n, 1}=o_{p}\left(m^{-1 / 2}\right)+O_{p}\left((m / n)^{2} m^{-1 / 2}\right) .
$$

Note that $\mathrm{E}\left(S_{n, 2}\right)=1$. Write

$$
m^{1 / 2}\left(S_{n, 2}-\mathrm{E}\left(S_{n, 2}\right)\right)=m^{1 / 2} n^{-1} \sum_{k=1}^{n}\left(\varepsilon_{k}^{2}-1\right)+\sum_{t=2}^{n} z_{t}
$$

where $z_{t}:=\varepsilon_{t} \sum_{s=1}^{t-1} \varepsilon_{s} d_{t-s}$ and $d_{s}:=2 m^{-1 / 2} n^{-1} \sum_{s=1}^{m} \cos \left(s \lambda_{j}\right)$. Note that

$$
m^{1 / 2} n^{-1} \sum_{k=1}^{n}\left(\varepsilon_{k}^{2}-1\right)=m^{1 / 2} n^{-1} O_{p}\left(n^{1 / 2}\right)=o_{p}(1) .
$$

On the other hand, the variables $\left\{z_{t}\right\}_{t=2}^{n}$ form a sequence of martingale differences and, using same argument as checking conditions (4.12) and (4.13) of the martingale central limit theorem in Robinson (1995b), it follows that $\sum_{t=2}^{n} z_{t} \stackrel{d}{\rightarrow} \mathrm{N}(0,1)$. Therefore

$$
m^{1 / 2}\left(S_{n, 2}-\mathrm{E}\left(S_{n, 2}\right)\right) \stackrel{d}{\longrightarrow} \mathrm{N}(0,1)
$$

which, together with (A.11), proves (A.9).

Next, we prove (3.2). By (3.4), $\widehat{d}-d=O_{p}\left(m_{w}^{-1 / 2}\right)$. The mean value theorem implies that

$$
\widehat{s}_{m}^{2}(\widehat{d})=\widehat{s}_{m}^{2}(d)+Q_{n},
$$


where

$$
Q_{n}:=(\widehat{d}-d) \frac{\partial}{\partial d} \widehat{s}_{m}^{2}(\widetilde{d}), \quad \widetilde{d} \in(\widehat{d}, d)
$$

and

$$
|\widetilde{d}-d| \leq|\widehat{d}-d|=o_{p}(1 / \log n)
$$

We have that

$$
\frac{\partial}{\partial d} \widehat{s}_{m}^{2}(\widetilde{d})=\frac{\partial}{\partial d}\left[p(\widetilde{d}) \widehat{b}_{m}(\widetilde{d})\right]=p^{\prime}(\widetilde{d}) \widehat{b}_{m}(\widetilde{d})+p(\widetilde{d}) \frac{\partial}{\partial d} \widehat{b}_{m}(\widetilde{d}) .
$$

Note that

$$
p(\widetilde{d}) \stackrel{d}{\rightarrow} p(d), \quad p^{\prime}(\widetilde{d}) \stackrel{d}{\rightarrow} p^{\prime}(d)
$$

whereas

$$
\frac{\partial}{\partial d} \widehat{b}_{m}(\widetilde{d})=2 m^{-1} \sum_{j=1}^{m} \lambda_{j}^{2 \widetilde{d}} \log \left(\lambda_{j}\right) I_{n}\left(\lambda_{j}\right)=2\left(\log \left(\lambda_{q}\right) D_{n, 1}+D_{n, 2}\right)
$$

where

$$
D_{n, 1}:=m^{-1} \sum_{j=1}^{m} \lambda_{j}^{2 \widetilde{d}} I_{n}\left(\lambda_{j}\right), \quad D_{n, 2}:=m^{-1} \sum_{j=1}^{m} \lambda_{j}^{2 \widetilde{d}} \log (j / q) I_{n}\left(\lambda_{j}\right) .
$$

By (A.13) and Lemma 6.2 of Dalla et al. (2006) it follows that

$$
D_{n, 1} \stackrel{p}{\rightarrow} b_{0}, \quad D_{n, 2} \stackrel{p}{\rightarrow} b_{0} \int_{0}^{1} \log x \mathrm{~d} x=-b_{0}, \quad \widehat{b}_{m}(\widetilde{d}) \stackrel{p}{\longrightarrow} b_{0} .
$$

Thus,

$$
\begin{aligned}
Q_{n} & =(\widehat{d}-d)\left[p^{\prime}(d) b_{0}+2 \log \left(\lambda_{m}\right) p(d) b_{0}-2 p(d) b_{0}\right]\left(1+o_{p}(1)\right) \\
& =2(\widehat{d}-d) \log \left(\lambda_{m}\right) s_{X}^{2}\left(1+o_{p}(1)\right)
\end{aligned}
$$

Equation (A.12), together with (A.9) and (A.14), implies (3.2).

\section{A.3 Auxiliary lemmas}

Set

$$
\eta_{n}(\lambda):=2 \pi b_{n}(\lambda) f(\lambda)
$$

where $b_{n}(\lambda)$ is an even real function and $f$ is the spectral density of $\left\{X_{t}\right\}$. Defining

$$
D_{n}(\lambda):=\sum_{t=1}^{n} \mathrm{e}^{\mathrm{i} t \lambda}=\mathrm{e}^{\mathrm{i}(n+1) \lambda / 2} \frac{\sin (n \lambda / 2)}{\sin (\lambda / 2)}
$$


we have

$$
\left|D_{n}(\lambda)\right| \leq C n(1+|n \lambda|)^{-1}, \quad|\lambda| \leq 3 \pi / 2 .
$$

Set $p_{n}:=(n / q)^{\epsilon}$ where $\epsilon>0$ is a small number. Then,

$$
q p_{n} / n=(q / n)^{1-\epsilon} \rightarrow 0
$$

Lemma A.1. Let $d \in(-1 / 2,1 / 4)$ and $F_{n}(u):=\int_{-\pi}^{\pi} \eta_{n}(u-x) \eta_{n}(x) \mathrm{d} x$. Under the assumptions of Theorem 2.1,

$$
\begin{gathered}
F_{n}(u) \leq F_{n}(0), \\
F_{n}(0) \sim q 4 \pi^{2} b_{0}^{2} \int_{-\infty}^{\infty}\left(\frac{\sin (x / 2)}{x / 2}\right)^{4}|x|^{-4 d} \mathrm{~d} x, \quad n \rightarrow \infty,
\end{gathered}
$$

and, for $p_{n}$ satisfying (A.18),

$$
\sup _{|u| \leq p_{n} / n}\left|F_{n}(u)-F_{n}(0)\right|=o(q) .
$$

Proof of Lemma A.1. By Cauchy's inequality,

$$
F_{n}(u) \leq\left(\int_{-\pi}^{\pi} \eta_{n}^{2}(u-x) \mathrm{d} x\right)^{1 / 2}\left(\int_{-\pi}^{\pi} \eta_{n}^{2}(x) \mathrm{d} x\right)^{1 / 2}=\int_{-\pi}^{\pi} \eta_{n}^{2}(x) \mathrm{d} x=F_{n}(0),
$$

since $\eta_{n}(x+2 \pi)=\eta_{n}(x)$, proving (A.19).

Using the asymptotic approximation $f(\lambda)=b_{0}|\lambda|^{-2 d}(1+o(1))$ together with (A.15) and (A.3), we get as $n \rightarrow \infty$

$$
\begin{aligned}
F_{n}(0) & =\int_{-\pi}^{\pi} \eta_{n}^{2}(x) \mathrm{d} x=4 \pi^{2} \int_{-\pi}^{\pi} q^{-2-4 d}\left(\frac{\sin (q x / 2)}{\sin (x / 2)}\right)^{4} f^{2}(x) \mathrm{d} x \\
& \sim q 4 \pi^{2} b_{0}^{2} \int_{-\infty}^{\infty}\left(\frac{\sin (x / 2)}{x / 2}\right)^{4}|x|^{-4 d} \mathrm{~d} x,
\end{aligned}
$$

proving (A.20).

By (A.15) and (A.3),

$$
\eta_{n}(\lambda)=2 \pi b_{n}(\lambda) f(\lambda)=2 \pi q^{-1-2 d}\left(\frac{\sin (q \lambda / 2)}{\sin (\lambda / 2)}\right)^{2}|\lambda|^{-2 d} g(\lambda) .
$$

Let $u_{n}$ maximize $q^{-1}\left|F_{n}(u)-F_{n}(0)\right|$ in the set $\left\{u:|u| \leq p_{n} / n\right\}$. Then,

$$
\begin{aligned}
\sup _{|u| \leq p_{n} / n} q^{-1}\left|F_{n}(u)-F_{n}(0)\right| & \leq \int_{-\pi}^{\pi} q^{-1}\left|\eta_{n}\left(u_{n}-x\right)-\eta_{n}(-x)\right| \eta_{n}(x) \mathrm{d} x \\
& \leq \int_{-\pi q}^{\pi q} q^{-2}\left|\eta_{n}\left(u_{n}-x q^{-1}\right)-\eta_{n}\left(-x q^{-1}\right)\right| \eta_{n}\left(x q^{-1}\right) \mathrm{d} x \\
& =\int_{2 q\left|u_{n}\right| \leq|x| \leq \pi q}+\int_{0 \leq|x|<2 q\left|u_{n}\right|}=: q_{n, 1}+q_{n, 2} .
\end{aligned}
$$


To prove (A.21), it remains show that

$$
q_{n, j} \rightarrow 0, \quad n \rightarrow \infty, \quad j=1,2 .
$$

We first show (A.22) for $j=1$. Observe that, if $2 q|u| \leq|x|$, then

$$
|x / q+u| \leq|x / q|+|u| \leq 2|x| / q, \quad|x / q+u| \geq|x / q|-|u| \geq|x| / 2 q .
$$

This, together with (1.4), (A.15), and (A.17), implies the bound

$$
\left|q^{-1} \eta_{n}\left(u_{n}-x q^{-1}\right)\right| \leq C(1+|x|)^{-2}|x|^{-2 d}=: M(x),
$$

which holds for all $|x| \in\left[2 q\left|u_{n}\right|, \pi q\right]$. Moreover, for any fixed $x$, as $n \rightarrow \infty$,

$$
q^{-1} \eta_{n}\left(u_{n}-x q^{-1}\right) \rightarrow 2 \pi\left(\frac{\sin (x / 2)}{x / 2}\right)^{2} b_{0}|x|^{-2 d} \leq C M(x)
$$

since $\sin ^{2}(x / 2) /(x / 2)^{2} \leq C(1+|x|)^{-2}$. Therefore, estimating $q^{-1}\left|\eta_{n}\left(u_{n}-x q^{-1}\right)\right| \leq$ $M(x), q^{-1}\left|\eta_{n}\left(-x q^{-1}\right)\right| \leq M(x)$, and $q^{-1}\left|\eta_{n}\left(x q^{-1}\right)\right| \leq M(x)$, it follows that

$$
q^{-2}\left|\eta_{n}\left(u_{n}-x q^{-1}\right)-\eta_{n}\left(-x q^{-1}\right)\right| \eta_{n}\left(x q^{-1}\right) \leq(M(x))^{2}
$$

and, for any fixed $x$,

$$
q^{-2}\left|\eta_{n}\left(u_{n}-x q^{-1}\right)-\eta_{n}\left(-x q^{-1}\right)\right| \eta_{n}\left(x q^{-1}\right) \rightarrow 0, \quad n \rightarrow \infty .
$$

Since $M(x)^{2}$ is an integrable function, the theorem of majorating convergence implies that $q_{n, 1} \rightarrow 0$.

To work out $q_{n, 2}$, note that in $q_{n, 2}$ we integrate over $|x| \leq 2 q\left|u_{n}\right| \leq 2 q p_{n} / n=$ : $t_{n} \rightarrow 0$, as $n \rightarrow \infty$. By (A.17), $q^{-1}\left|D_{q}\left(x / q+u_{n}\right)\right| \leq C$ and by (1.4), $q^{-2 d} f(x / q+$ $\left.u_{n}\right) \leq C\left|x+u_{n} q\right|^{-2 d}$. Therefore,

$$
q_{n, 2} \leq C \int_{0 \leq|x| \leq 2 q\left|u_{n}\right|}\left(\left|x+u_{n} q\right|^{-4 d}+|x|^{-4 d}\right) \mathrm{d} x \leq C \int_{0 \leq|x| \leq 2 t_{n}}|x|^{-4 d} \mathrm{~d} x \rightarrow 0
$$

as $n \rightarrow \infty$, since $4 d<1$ and $t_{n} \rightarrow 0$.

Lemma A.2. Let $d \in(1 / 4,1 / 2)$ and

$$
H_{n}(u):=\int_{-\pi}^{\pi} f(u-\lambda) b_{n}(\lambda) \mathrm{d} \lambda
$$

where $f(\lambda)$ is periodically extended to $\mathbb{R}$. Then, under the assumptions of Theorem 2.1,

$$
\sup _{|u| \leq p_{n}^{d} / n}\left|H_{n}(u)-H_{n}(0)\right|=o\left((q / n)^{1-2 d}\right)+o\left(q^{-1-2 d}\right)
$$

and

$$
\left|H_{n}(u)-H_{n}(0)\right| \leq C(|u| q)^{1-2 d}, \quad|u| \leq \pi .
$$


Proof of Lemma A.2. First, we show (A.24). Let $|u| \leq p_{n}^{d} / n$, where $p_{n} \rightarrow \infty$ is the same as in (A.18). Then,

$$
\begin{aligned}
H_{n}(u)-H_{n}(0) & =q^{-1-2 d} \int_{-\pi}^{\pi}(f(u-x)-f(-x))\left|D_{q}(x)\right|^{2} \mathrm{~d} x \\
& =\int_{0 \leq|x|<2 p_{n} / n}+\int_{2 p_{n} / n \leq|x| \leq 1}+\int_{1 \leq|x| \leq \pi} \\
& =: i_{n, 1}+i_{n, 2}+i_{n, 3} .
\end{aligned}
$$

To work out $i_{n, 1}$, note that if $|x|<2 p_{n} / n$, then $|x| q \leq 2 p_{n} q / n=o(1)$ and therefore

$$
\begin{aligned}
\left|D_{q}(x)\right|^{2} & =\left(\frac{\sin (q x / 2)}{\sin (x / 2)}\right)^{2}=\frac{(q x / 2)^{2}\left(1+O(q x)^{2}\right)^{2}}{(x / 2)^{2}\left(1+O(x)^{2}\right)^{2}} \\
& =q^{2}\left(1+O(q x)^{2}\right)=q^{2}\left(1+O\left(\left(q p_{n} / n\right)^{2}\right)\right)
\end{aligned}
$$

for all $x \in[-\pi, \pi]$. Since $|u| \leq p_{n}^{d} / n$ and $f(x) \leq C|x|^{-2 d}$, we obtain

$$
\begin{aligned}
\left|i_{n, 1}\right| & =q^{1-2 d}\left|\int_{-2 p_{n} / n}^{2 p_{n} / n}(f(x-u)-f(x))\left(1+O\left(\left(q p_{n} / n\right)^{2}\right)\right) \mathrm{d} x\right| \\
& \leq q^{1-2 d}\left[2 \int_{2 p_{n} / n-|u|}^{2 p_{n} / n+|u|} f(x) \mathrm{d} x+O\left(\left(q p_{n} / n\right)^{2}\right) \int_{-2 p_{n} / n-|u|}^{2 p_{n} / n+|u|} f(x) \mathrm{d} x\right] \\
& \leq C q^{1-2 d}\left[|u|\left(p_{n} / n\right)^{-2 d}+O\left(\left(q p_{n} / n\right)^{2}\right) \int_{-3 p_{n} / n}^{3 p_{n} / n}|x|^{-2 d} \mathrm{~d} x\right] \\
& \leq C(q / n)^{1-2 d} p_{n}^{-d}+(q / n)^{1-2 d} p_{n}^{3-2 d}(q / n)^{2}=o\left((q / n)^{1-2 d}\right) .
\end{aligned}
$$

To work out $i_{n, 2}$, observe that if $x \in\left[2 p_{n} / n, 1\right]$ and $|u| \leq p_{n}^{d} / n$, then $|x-u| \geq$ $|x|-|u| \geq|x| / 2$, as $n \rightarrow \infty$, and therefore

$$
\begin{aligned}
& |f(u-x)-f(-x)| \\
\leq & || x-\left.u\right|^{-2 d}-\left.|x|^{-2 d}|g(x-u)+| x\right|^{-2 d}|g(x-u)-g(x)| \leq C|u||x|^{-2 d-1},
\end{aligned}
$$

since, by the mean value theorem,

$$
|| x-\left.u\right|^{-2 d}-\left.|x|^{-2 d}|\leq C| u\left|\sup _{|x-u| \leq y \leq|x|}\right| y\right|^{-2 d-1} \leq C|u||x|^{-2 d-1},
$$

and $|\mathrm{d} g(x) / \mathrm{d} x| \leq C$ by the assumption of Theorem 2.1(b). Using

$$
\left|D_{q}(x)\right|^{2} \leq q^{2}
$$

and $|u| \leq p_{n}^{d} / n$, we obtain that, for $|u| \leq p_{n}^{d} / n$,

$$
\left|i_{n, 2}\right| \leq q^{1-2 d}|u| \int_{p_{n} / n}^{1}|x|^{-2 d-1} \mathrm{~d} x \leq C(q / n)^{1-2 d} p_{n}^{-d}=o\left((q / n)^{1-2 d}\right) .
$$


Finally, for $1 \leq|x| \leq \pi$, (A.17) implies that $\left|D_{q}(x)\right|^{2} \leq C$, and therefore

$$
\left|i_{n, 3}\right| \leq q^{-1-2 d} \int_{1 \leq|x| \leq \pi}|f(x-u)-f(x)| \mathrm{d} x=o\left(q^{-1-2 d}\right)
$$

for all $|u| \leq p_{n}^{d} / n \rightarrow 0$ as $n \rightarrow \infty$. Thus,

$$
\sup _{|u| \leq p_{n}^{d} / n}\left|H_{n}(u)-H_{n}(0)\right|=o\left((q / n)^{1-2 d}\right)+o\left(q^{-1-2 d}\right),
$$

to complete proof of (A.24).

Now we show (A.25). Applying in (A.26) the result (A.28) and, for $2|u| \leq|x|$, using the result (A.27), we obtain

$$
\begin{aligned}
\left|H_{n}(u)-H_{n}(0)\right| & \leq C q^{1-2 d} \int_{-\pi}^{\pi}|f(u-x)-f(-x)| \mathrm{d} x \\
& \leq C q^{1-2 d}\left(\int_{2|u| \leq|x| \leq \pi}|u||x|^{-2 d-1} \mathrm{~d} x+\int_{|x| \leq 2|u|}\left(|x-u|^{-2 d}+|x|^{-2 d}\right) \mathrm{d} x\right) \\
& \leq C(|u| q)^{1-2 d},
\end{aligned}
$$

to prove (A.25).

Lemma A.3. Let $-1<\gamma<1$. Then for any $\epsilon>0, x \in \mathbb{R}$ and $n \in \mathbb{Z}$,

$$
J_{n}(\gamma):=\int_{-\pi}^{\pi}|\lambda|^{-\gamma}\left|D_{n}(\lambda) D_{n}(\lambda+x)\right| \mathrm{d} \lambda \leq C n^{1+\gamma}(1+|n x|)^{-1-\min \{0, \gamma\}+\epsilon} .
$$

Proof of Lemma A.3. It suffices to show that (A.29) holds when $\epsilon>0$ is sufficiently small. Write

$$
J_{n}(\gamma):=\int_{|\lambda| \leq \pi / 2}+\int_{\pi / 2<|\lambda| \leq \pi}=: J_{n, 1}(\gamma)+J_{n, 2}(\gamma)
$$

Using (A.17), we obtain that

$$
\begin{aligned}
J_{n, 1}(\gamma) & \leq C \int_{-\pi / 2}^{\pi / 2}|\lambda|^{-\gamma} \frac{n}{1+|n \lambda|} \frac{n}{1+|n(\lambda+x)|} \mathrm{d} \lambda \\
& \leq C n^{1+\gamma} \int_{-\infty}^{\infty}|\lambda|^{-\gamma} \frac{1}{1+|\lambda|} \frac{1}{1+|\lambda+n x|} \mathrm{d} \lambda
\end{aligned}
$$

First, we prove that (A.29) holds for $|n x| \geq 1$.

a) Let $\gamma \in[0,1)$ and choose $\epsilon>0$ such that $\gamma<1-\epsilon / 2$. Then, by (A.30),

$$
\begin{aligned}
J_{n, 1}(\gamma) & \leq C n^{1+\gamma} \int_{-\infty}^{\infty}|\lambda|^{-1+\epsilon / 2}|\lambda+n x|^{-1+\epsilon / 2} \mathrm{~d} \lambda \\
& \leq C n^{1+\gamma}|n x|^{-1+\epsilon} \int_{-\infty}^{\infty}|\lambda|^{-1+\epsilon / 2}|\lambda+1|^{-1+\epsilon / 2} \mathrm{~d} \lambda \leq C n^{1+\gamma}(1+|n x|)^{-1+\epsilon}
\end{aligned}
$$


which proves (A.29).

b) Let $\gamma \in(-1,0)$ and choose $\epsilon>0$ such that $\gamma+1-\epsilon>0$. Then, by (A.30),

$$
\begin{aligned}
J_{n, 1}(\gamma) & \leq C n^{1+\gamma} \int_{-\infty}^{\infty}|\lambda|^{-\gamma-1}|\lambda+n x|^{-1+\epsilon} \mathrm{d} \lambda \\
& \leq C n^{1+\gamma}|n x|^{-1-\gamma+\epsilon} \int_{-\infty}^{\infty}|\lambda|^{-\gamma-1}|\lambda+1|^{-1+\epsilon} \mathrm{d} \lambda \leq C n^{1+\gamma}(1+|n x|)^{-1-\gamma+\epsilon},
\end{aligned}
$$

which implies (A.29) .

If $|n x| \leq 1$, then applying Cauchy's inequality in (A.30) we obtain that

$$
\begin{aligned}
J_{n, 1}(\gamma) & \leq C n^{1+\gamma}\left(\int_{-\infty}^{\infty}|\lambda|^{-\gamma}(1+|\lambda|)^{-2} \mathrm{~d} \lambda\right)^{1 / 2}\left(\int_{-\infty}^{\infty}|\lambda|^{-\gamma}(1+|\lambda+n x|)^{-2} \mathrm{~d} \lambda\right)^{1 / 2} \\
& \leq C n^{1+\gamma} \leq C n^{1+\gamma}(1+|n x|)^{-1-\min \{0, \gamma\}+\epsilon}
\end{aligned}
$$

proving (A.29).

If $\pi / 2 \leq|\lambda| \leq \pi$, then $|\lambda|^{-\gamma}\left|D_{n}(\lambda)\right| \leq C$, by (A.17), and therefore

$$
J_{n, 2}(\gamma) \leq C \int_{-\pi}^{\pi}\left|D_{n}(\lambda+x)\right| \mathrm{d} \lambda \leq C \int_{-2 \pi}^{2 \pi}\left|D_{n}(\lambda)\right| \mathrm{d} \lambda \leq C \log n .
$$

Observing that

$$
n^{1+\gamma}(1+|n x|)^{-1-\min \{0, \gamma\}+\epsilon} \geq n^{1+\gamma}(1+|n \pi|)^{-1-\min \{0, \gamma\}+\epsilon} \geq n^{\epsilon},
$$

this implies that $J_{n, 2}(\gamma)$ satisfies (A.29).

Lemma A.4. Under the assumptions of Theorem 2.1, $R_{n}:=\int_{-\pi}^{\pi} b_{n}(\lambda)\left(\bar{I}_{n}(\lambda)-\right.$ $\left.I_{n}(\lambda)\right) \mathrm{d} \lambda$ satisfies

$$
\mathrm{E}\left(\left|R_{n}\right|\right) \leq C\left[(q / n)^{1-2 d}+(q / n)\right] .
$$

Proof of Lemma A.4. Denote

$$
w_{n}(\lambda):=(2 \pi n)^{-1 / 2} \sum_{t=1}^{n} \mathrm{e}^{\mathrm{i} t \lambda}\left(X_{t}-\mu\right) .
$$

Then,

$$
\begin{aligned}
I_{n}(\lambda) & =\left|w_{n}(\lambda)+(\mu-\bar{X})(2 \pi n)^{-1 / 2} D_{n}(\lambda)\right|^{2} \\
& =I_{n}(\lambda)+(\mu-\bar{X})(2 \pi n)^{-1 / 2}\left[w_{n}(\lambda) D_{n}(-\lambda)+w_{n}(-\lambda) D_{n}(\lambda)\right] \\
& +(\mu-\bar{X})^{2}(2 \pi n)^{-1}\left|D_{n}(\lambda)\right|^{2}
\end{aligned}
$$

Therefore,

$$
\left|R_{n}\right| \leq C\left(|\mu-\bar{X}| n^{-1 / 2}\left|T_{n}\right|+(\mu-\bar{X})^{2} n^{-1} \int_{-\pi}^{\pi} b_{n}(\lambda)\left|D_{n}(\lambda)\right|^{2} \mathrm{~d} \lambda\right),
$$


where

$$
T_{n}:=\int_{-\pi}^{\pi} b_{n}(\lambda) w_{n}(\lambda) D_{n}(-\lambda) \mathrm{d} \lambda .
$$

By (1.2), $\mathrm{E}\left((\mu-\bar{X})^{2}\right) \leq C n^{-1+2 d}$. We shall show below that

$$
\mathrm{E}\left(\left|T_{n}\right|^{2}\right) \leq C \begin{cases}q^{2-4 d} n^{2 d}, & \text { if } 0 \leq d<1 / 2, \\ q^{2} n^{-2 d}, & \text { if }-1 / 2<d<0 .\end{cases}
$$

To work out the last term in (A.32), observe that, since $b_{n}(\lambda) \leq C q^{1-2 d}$, then

$$
n^{-1} \int_{-\pi}^{\pi} b_{n}(\lambda)\left|D_{n}(\lambda)\right|^{2} \mathrm{~d} \lambda \leq C n^{-1} q^{1-2 d} \int_{-\pi}^{\pi}\left|D_{n}(\lambda)\right|^{2} \mathrm{~d} \lambda \leq C q^{1-2 d},
$$

because

$$
\int_{-\pi}^{\pi}\left|D_{n}(\lambda)\right|^{2} \mathrm{~d} \lambda=2 \pi n
$$

Therefore,

$$
\begin{aligned}
\mathrm{E}\left(\left|R_{n}\right|\right) & \leq C\left(\mathrm{E}\left((\mu-\bar{X})^{2}\right)\right)^{1 / 2} n^{-1 / 2}\left(\mathrm{E}\left(\left|T_{n}\right|^{2}\right)\right)^{1 / 2}+\mathrm{E}\left((\mu-\bar{X})^{2}\right) O\left(q^{1-2 d}\right) \\
& \leq C n^{-1+d}\left(\mathrm{E}\left(\left|T_{n}\right|^{2}\right)\right)^{1 / 2}+O\left((q / n)^{1-2 d}\right) .
\end{aligned}
$$

Applying (A.33), we see that for $d \geq 0, \mathrm{E}\left(\left|R_{n}\right|\right) \leq C(q / n)^{1-2 d}$; whereas for $d<0$, $\mathrm{E}\left(\left|R_{n}\right|\right) \leq C\left((q / n)+(q / n)^{1-2 d}\right) \leq C(q / n)$; proving (A.31).

It remains to show (A.33). Note that

$$
\begin{aligned}
\mathrm{E}\left(w_{n}\left(\lambda_{1}\right) w_{n}\left(-\lambda_{2}\right)\right) & =(2 \pi n)^{-1} \sum_{t, s=1}^{n} \mathrm{e}^{\mathrm{i}\left(t \lambda_{1}-s \lambda_{2}\right)} \int_{-\pi}^{\pi} \mathrm{e}^{\mathrm{i}(t-s) x} f(x) \mathrm{d} x \\
& =(2 \pi n)^{-1} \int_{-\pi}^{\pi} D_{n}\left(\lambda_{1}+x\right) D_{n}\left(-\lambda_{2}-x\right) f(x) \mathrm{d} x .
\end{aligned}
$$

Thus,

$$
\begin{aligned}
\mathrm{E}\left(\left|T_{n}\right|^{2}\right)= & (2 \pi n)^{-1} \int_{-\pi}^{\pi}\left(\int_{-\pi}^{\pi} \int_{-\pi}^{\pi} b_{n}\left(\lambda_{1}\right) D_{n}\left(-\lambda_{1}\right) b_{n}\left(\lambda_{2}\right) D_{n}\left(-\lambda_{2}\right)\right. \\
& \left.\times D_{n}\left(\lambda_{1}+x\right) D_{n}\left(-\lambda_{2}-x\right) \mathrm{d} \lambda_{1} \mathrm{~d} \lambda_{2}\right) f(x) \mathrm{d} x \\
= & (2 \pi n)^{-1} \int_{-\pi}^{\pi}\left|\int_{-\pi}^{\pi} b_{n}(\lambda) D_{n}(-\lambda) D_{n}(\lambda+x) \mathrm{d} \lambda\right|^{2} f(x) \mathrm{d} x .
\end{aligned}
$$

First, let $0 \leq d<1 / 2$. Note that $b_{n}(\lambda) \leq C q^{1-2 d}$ for $d \geq 0$, and $f(x) \leq C|x|^{-2 d}$ by (1.4). Therefore, applying to (A.34) result (A.29) with $\gamma=0$ and $0<\epsilon<1 / 2$, we obtain that

$$
\mathrm{E}\left(\left|T_{n}\right|^{2}\right) \leq n q^{2-4 d} \int_{-\pi}^{\pi}(1+|n x|)^{-2+2 \epsilon}|x|^{-2 d} \mathrm{~d} x
$$




$$
\leq C q^{2-4 d} n^{2 d} \int_{-\infty}^{\infty}(1+|x|)^{-2+2 \epsilon}|x|^{-2 d} \mathrm{~d} x \leq C q^{2-4 d} n^{2 d}
$$

proving (A.33).

Second, let $-1 / 2<d<0$. Then,

$$
b_{n}(\lambda) \leq C q^{-1-2 d}\left|\frac{\sin (q \lambda / 2)}{\lambda}\right|^{2+2 \delta}\left|\frac{\sin (q \lambda / 2)}{\lambda}\right|^{-2 d} \leq C q|\lambda|^{2 d}
$$

since $|\sin (q \lambda / 2) / \lambda| \leq C q,|\lambda| \leq \pi$. Applying to (A.34) result (A.29) with $\gamma=-2 d$ and $\epsilon>0$ such that $2+2 d-2 \epsilon>1$, we obtain that

$$
\mathrm{E}\left(\left|T_{n}\right|^{2}\right) \leq n^{1-4 d} q^{2} \int_{-\pi}^{\pi}(1+|n x|)^{-2-2 \gamma+2 \epsilon}|x|^{-2 d} \mathrm{~d} x \leq C n^{-2 d} q^{2},
$$

to complete the proof of (A.33).

\section{A.4 Propositions}

Proposition A.1. Let the assumptions of Theorem 2.1 be satisfied and $-1 / 2<d<$ 1/4. Then,

$$
(n / q)^{1 / 2}\left(\int_{-\pi}^{\pi} b_{n}(\lambda) I_{n}(\lambda) \mathrm{d} \lambda-\int_{-\pi}^{\pi} b_{n}(\lambda) f(\lambda) \mathrm{d} \lambda\right) \stackrel{d}{\longrightarrow} \mathrm{N}\left(0, v_{d}^{2}\right),
$$

where $v_{d}^{2}$ is given by (2.11).

Proof of Proposition A.1. Define the $n \times n$ matrix $\boldsymbol{E}_{n}:=\left(e_{t-s}\right)_{t, s=1}$ with entries $e_{t}:=\int_{-\pi}^{\pi} \mathrm{e}^{\mathrm{i} t \lambda} \eta_{n}(\lambda) \mathrm{d} \lambda$ and $t \in \mathbb{Z}$, and denote its Euclidean norm by $\left\|\boldsymbol{E}_{n}\right\|:=$ $\left(\sum_{t, s=1}^{n} e_{t-s}^{2}\right)^{1 / 2}$. If

$$
\left|b_{n}(\lambda)\right| \leq k_{n}|\lambda|^{2 d-\delta}, \quad|\lambda| \leq \pi
$$

where $0 \leq \delta<1 / 4, k_{n} \geq 0$ and, as $n \rightarrow \infty$,

$$
\frac{k_{n} n^{\max \{\delta, d, 0\}} \log n}{\left\|\boldsymbol{E}_{n}\right\|} \rightarrow 0
$$

and

$$
\int_{-\pi}^{\pi} \eta_{n}(\lambda) \mathrm{d} \lambda=o\left(n^{-1 / 2}\left\|\boldsymbol{E}_{n}\right\|\right)
$$

where $\eta_{n}(\lambda)$ is defined by (A.15), then Corollary 1.2 of Bhansali, Giraitis and Kokoszka (2004) implies that

$$
\frac{\sqrt{2} \pi n}{\left\|\boldsymbol{E}_{n}\right\|}\left(\int_{-\pi}^{\pi} b_{n}(\lambda) I_{n}(\lambda) \mathrm{d} \lambda-\int_{-\pi}^{\pi} b_{n}(\lambda) f(\lambda) \mathrm{d} \lambda\right) \stackrel{d}{\rightarrow} \mathrm{N}(0,1) .
$$


Therefore, to verify convergence (A.36), it suffices to check that conditions (A.37)(A.39) are satisfied in the case of the function $b_{n}(\lambda)$, defined in equation (A.3). Suppose that the asymptotic approximation

$$
\left\|\boldsymbol{E}_{n}\right\|^{2} \sim q n 8 \pi^{3} b_{0}^{2} \int_{-\infty}^{\infty}\left(\frac{\sin (x / 2)}{x / 2}\right)^{4}|x|^{-4 d} \mathrm{~d} x=q n 2 \pi^{2} v_{d}^{2},
$$

is valid for $d \in(-1 / 2,1 / 4)$. We shall prove this at the end of the proposition, but we first show that it implies the required (A.36).

Assume that $d \geq 0$. Then

$$
b_{n}(\lambda) \leq C q^{1-2 d}=: C k_{n},
$$

and therefore $b_{n}(\lambda)$ has property (A.37) with $k_{n}=q^{1-2 d}$ and $\delta=2 d$. Then

$$
\frac{k_{n} n^{\max \{\delta, d, 0\}} \log n}{\left\|\boldsymbol{E}_{n}\right\|} \leq C \frac{q^{1-2 d} n^{2 d} \log n}{(q n)^{1 / 2}} \leq C(q / n)^{1 / 2-2 d} \log n \rightarrow 0,
$$

since $d<1 / 4$ and $q$ satisfies (2.3). Thus (A.38) holds. On the other hand, from the definitions of $b_{n}, \eta_{n}(\lambda)$ and assumption (1.4), it follows that

$$
\begin{aligned}
\int_{-\pi}^{\pi}\left|\eta_{n}(\lambda)\right| \mathrm{d} \lambda & \leq C \int_{-\pi}^{\pi} q^{-1-2 d}\left(\frac{\sin (q \lambda / 2)}{\sin (\lambda / 2)}\right)^{2}|\lambda|^{-2 d} \mathrm{~d} \lambda \\
& \leq C \int_{-\infty}^{\infty}\left(\frac{\sin (\lambda / 2)}{\lambda / 2}\right)^{2}|\lambda|^{-2 d} \mathrm{~d} \lambda \leq C
\end{aligned}
$$

which, together with (A.41), implies (A.39). Hence assumptions (A.37)-(A.39) are satisfied and (A.40), together with (A.41), implies (A.36).

Assume now that $-1 / 2<d<0$. Then, (A.35) shows that (A.37) holds with $k_{n}=q$ and $\delta=0$. To check (A.38), observe that

$$
\frac{k_{n} n^{\max \{\delta, d, 0\}} \log n}{\left\|\boldsymbol{E}_{n}\right\|} \leq C \frac{q \log n}{(q n)^{1 / 2}} \leq C(q / n)^{1 / 2} \log n \rightarrow 0,
$$

by (A.41) and (2.3), whereas (A.39) follows from (A.43) and (A.41). Hence, the assumptions (A.37)-(A.39) are satisfied, and (A.36) follows from (A.40) and (A.41).

It remains to show (A.41). Write

$$
\begin{aligned}
\left\|\boldsymbol{E}_{n}\right\|^{2} & =\sum_{t, s=1}^{n} e_{t-s}^{2}=\int_{-\pi}^{\pi} \int_{-\pi}^{\pi}\left|\sum_{t=1}^{n} \mathrm{e}^{\mathrm{i} t(x+y)}\right|^{2} \eta_{n}(x) \eta_{n}(y) \mathrm{d} x \mathrm{~d} y \\
& =\int_{-\pi}^{\pi}\left|\sum_{t=1}^{n} \mathrm{e}^{\mathrm{i} t u}\right|^{2} F_{n}(u) \mathrm{d} u=\int_{-\pi}^{\pi}\left(\frac{\sin (u n / 2)}{\sin (u / 2)}\right)^{2} F_{n}(u) \mathrm{d} u
\end{aligned}
$$


where

$$
F_{n}(u)=\int_{-\pi}^{\pi} \eta_{n}(u-x) \eta_{n}(x) \mathrm{d} x
$$

and $\eta_{n}(x)$ is periodically extended to $\mathbb{R}$. Then

$$
\left\|\boldsymbol{E}_{n}\right\|^{2}=\int_{-\pi}^{\pi}\left|\sum_{t=1}^{n} \mathrm{e}^{\mathrm{i} t u}\right|^{2} F_{n}(0) \mathrm{d} u+\int_{-\pi}^{\pi}\left|\sum_{t=1}^{n} \mathrm{e}^{\mathrm{i} t u}\right|^{2}\left(F_{n}(u)-F_{n}(0)\right) \mathrm{d} u=: q_{n, 1}+q_{n, 2} .
$$

By (A.20),

$$
v_{n, 1}=2 \pi n F_{n}(0) \sim 16 q n \pi^{3} b_{0}^{2} \int_{0}^{\infty}\left(\frac{\sin (x / 2)}{x / 2}\right)^{4} x^{-4 d} \mathrm{~d} x .
$$

Finally, we need to show that

$$
q_{n, 2}=o(q n) .
$$

By (A.18) and (A.21),

$$
\int_{|u| \leq p_{n} / n}\left|\sum_{t=1}^{n} \mathrm{e}^{\left.\mathrm{i} t u\right|^{2}}\right| F_{n}(u)-\left.F_{n}(0)\left|\mathrm{d} u=o(q) \int_{|u| \leq \pi}\right| \sum_{t=1}^{n} \mathrm{e}^{\mathrm{i} t u}\right|^{2} \mathrm{~d} u=o(q n),
$$

whereas by (A.19)-(A.20), $\left|F_{n}(u)-F_{n}(0)\right| \leq 2 F_{n}(0)=O(q)$, and therefore

$$
\int_{p_{n} / n \leq|u| \leq \pi}\left|\sum_{t=1}^{n} \mathrm{e}^{\mathrm{i} t u}\right|^{2}\left|F_{n}(u)-F_{n}(0)\right| \mathrm{d} u=o(q n),
$$

since

$$
\int_{p_{n} / n \leq|u| \leq \pi}\left(\frac{\sin (u n / 2)}{\sin (u / 2)}\right)^{2} \mathrm{~d} u \leq C n \int_{p_{n} \leq|u| \leq \infty}\left(\frac{\sin (u / 2)}{u / 2}\right)^{2} \mathrm{~d} u=o(n),
$$

because $p_{n} \rightarrow \infty$. This completes the proof (A.45).

Proposition A.2. Assume that the spectral density $f$ has the property

$$
f(\lambda)=b_{0}|\lambda|^{-2 d}\left(1+O\left(\lambda^{2}\right)\right), \quad \text { as } \lambda \rightarrow 0
$$

with $|d|<1 / 2$. Then, as $n \rightarrow \infty$,

$$
\int_{-\pi}^{\pi} b_{n}(\lambda) f(\lambda) \mathrm{d} \lambda=s_{X}^{2}+q^{-1-2 d} B+o\left(q^{-1-2 d}\right)
$$

where $B$ is given in (2.6).

proof of Proposition A.2. Set

$$
h_{1}(\lambda)=\frac{b_{0}|\lambda|^{-2 d}}{(\lambda / 2)^{2}}, \quad h_{2}(\lambda)=\frac{f(\lambda)}{\sin ^{2}(\lambda / 2)}-h_{1}(\lambda) .
$$


Then, we can write

$$
\begin{gathered}
i_{n}:=\int_{-\pi}^{\pi} b_{n}(\lambda) f(\lambda) \mathrm{d} \lambda=q^{-1-2 d} \int_{-\pi}^{\pi} \sin ^{2}(q \lambda / 2) \frac{f(\lambda)}{\sin ^{2}(\lambda / 2)} \mathrm{d} \lambda \\
=q^{-1-2 d} \int_{-\pi}^{\pi} \sin ^{2}(q \lambda / 2)\left(h_{1}(\lambda)+h_{2}(\lambda)\right) \mathrm{d} \lambda=q^{-1-2 d}\left(i_{n, 1}-2 i_{n, 2}+i_{n, 3}\right),
\end{gathered}
$$

where

$$
i_{n, 1}=\int_{-\infty}^{\infty} \sin ^{2}(q \lambda / 2) h_{1}(\lambda) \mathrm{d} \lambda=q^{1+2 d} \int_{-\infty}^{\infty} \sin ^{2}(\lambda / 2) \frac{b_{0}|\lambda|^{-2 d}}{(\lambda / 2)^{2}} \mathrm{~d} \lambda=q^{1+2 d} s_{X}^{2},
$$

and

$$
i_{n, 2}=\int_{\pi}^{\infty} \sin ^{2}(q \lambda / 2) h_{1}(\lambda) \mathrm{d} \lambda, \quad i_{n, 3}=\int_{-\pi}^{\pi} \sin ^{2}(q \lambda / 2) h_{2}(\lambda) \mathrm{d} \lambda .
$$

Since $\sin ^{2}(q \lambda / 2)=(1-\cos (q \lambda)) / 2$ and $\left|h_{1}\right|$ is an integrable function in $[\pi, \infty)$, then

$$
\begin{gathered}
i_{n, 2}=\int_{\pi}^{\infty} \sin ^{2}(q \lambda / 2) h_{1}(\lambda) \mathrm{d} \lambda=(1 / 2) \int_{\pi}^{\infty} h_{1}(\lambda) \mathrm{d} \lambda-(1 / 2) \int_{\pi}^{\infty} \cos (q \lambda) h_{1}(\lambda) \mathrm{d} \lambda \\
\rightarrow(1 / 2) \int_{\pi}^{\infty} h_{1}(\lambda) \mathrm{d} \lambda, \quad q \rightarrow \infty .
\end{gathered}
$$

Bearing in mind that by assumption (1.4), $f(\lambda)=b_{0}|\lambda|^{-2 d}\left(1+O\left(\lambda^{2}\right)\right)$ and $\sin (\lambda) / \lambda=1+O\left(\lambda^{2}\right)$, as $\lambda \rightarrow 0$, it follows that $\left|h_{2}(\lambda)\right| \leq C|\lambda|^{-2 d}$ for all $|\lambda| \leq \pi$. Since $|\lambda|^{-2 d}$ is an integrable function in $[-\pi, \pi]$, then

$$
\begin{gathered}
i_{n, 3}=\int_{-\pi}^{\pi} \sin ^{2}(q \lambda / 2) h_{2}(\lambda) \mathrm{d} \lambda=(1 / 2) \int_{-\pi}^{\pi} h_{1}(\lambda) \mathrm{d} \lambda-(1 / 2) \int_{-\pi}^{\pi} \cos (q \lambda) h_{2}(\lambda) \mathrm{d} \lambda \\
\rightarrow(1 / 2) \int_{-\pi}^{\pi} h_{2}(\lambda) \mathrm{d} \lambda, \quad q \rightarrow \infty .
\end{gathered}
$$

Estimates of $i_{n, j}, j=1,2,3$ imply (A.46).

Proposition A.3. Let the assumptions of Theorem 2.1 be satisfied and $1 / 4<d<$ 1/2. Define

$$
U_{n}:=q^{1-2 d} \int_{-\pi}^{\pi} I_{n}(\lambda) \mathrm{d} \lambda
$$

Then, as $n \rightarrow \infty$,

$$
\begin{gathered}
\operatorname{var}\left(\widetilde{s}_{q}^{2}(d)-U_{n}\right)=o\left((q / n)^{2-4 d}\right), \\
(n / q)^{1-2 d}\left(U_{n}-\mathrm{E}\left(U_{n}\right)\right) \stackrel{d}{\longrightarrow} 2 \pi b_{0} R(d),
\end{gathered}
$$

where $R(d)$ is given by (2.7), and

$$
\mathrm{E}\left(\widetilde{s}_{q}^{2}(d)\right)=s_{X}^{2}+q^{-1-2 d} B+o\left((q / n)^{1-2 d}+q^{-1-2 d}\right),
$$

where $B$ is given by $(2.6)$. 
Proof of Proposition A.3. Proof of (A.48). Set

$$
d_{n}\left(u_{1}, u_{2}\right):=\sum_{t, s=1}^{n} B_{n}(t-s) \psi_{t-u_{1}} \psi_{s-u_{2}},
$$

where $\psi_{t}$ are the coefficients of the linear process $X_{t}$,

$$
B_{n}(t):=\int_{-\pi}^{\pi} \mathrm{e}^{\mathrm{i} t x} \beta(x) \mathrm{d} x, \quad \beta(x)=\left|D_{q}(x)\right|^{2} q^{-2}-1,
$$

and $D_{q}(\lambda)$ is defined in (A.16). For simplicity, let $\psi_{t}=0, t \leq-1$. Then,

$$
\widetilde{s}_{q}^{2}(d)-U_{n}=\int_{-\pi}^{\pi}\left(b_{n}(\lambda)-q^{1-2 d}\right) I_{n}(\lambda) \mathrm{d} \lambda=q^{1-2 d}(2 \pi n)^{-1} \sum_{u_{1}, u_{2} \in \mathbb{Z}} d_{n}\left(u_{1}, u_{2}\right) \varepsilon_{u_{1}} \varepsilon_{u_{2}} .
$$

Note that if

$$
S=\sum_{u_{1}, u_{2} \in \mathbb{Z}} c\left(u_{1}, u_{2}\right)\left(\varepsilon_{u_{1}} \varepsilon_{u_{2}}-\mathrm{E}\left(\varepsilon_{u_{1}} \varepsilon_{u_{2}}\right)\right)
$$

is a quadratic form with real coefficients $c\left(u_{1}, u_{2}\right)$ where $\left\{\varepsilon_{t}\right\}$ is a sequence of i.i.d. random variables with zero mean and finite fourth moment, then

$$
\operatorname{var}(S) \leq C \sum_{u_{1}, u_{2} \in \mathbb{Z}} c^{2}\left(u_{1}, u_{2}\right) .
$$

Therefore, by (A.51),

$$
\operatorname{var}\left(\widetilde{s}_{q}^{2}(d)-U_{n}\right) \leq C q^{2-4 d} n^{-2} \sum_{u_{1}, u_{2} \in \mathbb{Z}}\left|d_{n}\left(u_{1}, u_{2}\right)\right|^{2}=: i_{n} .
$$

Since $\psi_{j}, j \in \mathbb{Z}$, are square summable by Assumption L, there exists function $\widehat{\psi}(x),|x| \leq \pi$, such that $\psi_{t}=\int_{-\pi}^{\pi} \mathrm{e}^{\mathrm{i} t x} \widehat{\psi}(x) \mathrm{d} x, t \in \mathbb{Z}$. Therefore,

$$
\begin{aligned}
d_{n}\left(u_{1}, u_{2}\right)= & \int_{-\pi}^{\pi} \int_{-\pi}^{\pi} \mathrm{e}^{-\mathrm{i} u_{1} x_{1}} \mathrm{e}^{-\mathrm{i} u_{1} x_{2}} \\
& \times\left[\mathrm{e}^{\mathrm{i} t x_{1}} \mathrm{e}^{\mathrm{i} s x_{2}} \widehat{\psi}\left(x_{1}\right) \widehat{\psi}\left(x_{2}\right) \int_{-\pi}^{\pi} \beta(y) D_{n}\left(y+x_{1}\right) D_{n}\left(-y+x_{2}\right) \mathrm{d} y\right] \mathrm{d} x_{1} \mathrm{~d} x_{2}
\end{aligned}
$$

for $u_{1}, u_{2} \in \mathbb{Z}$. By Parseval's equality,

$$
\begin{aligned}
i_{n}= & C q^{2-4 d} n^{-2} \int_{-\pi}^{\pi} \int_{-\pi}^{\pi} \mathrm{d} x_{1} \mathrm{~d} x_{2}\left|\widehat{\psi}\left(x_{1}\right)\right|^{2}\left|\widehat{\psi}\left(x_{2}\right)\right|^{2} \\
& \times\left|\int_{-\pi}^{\pi} \mathrm{d} y \beta(y) D_{n}\left(x_{1}+y\right) D_{n}\left(x_{2}-y\right)\right|^{2} .
\end{aligned}
$$

Set $I_{1}:=\left\{|y| \leq n^{\alpha-1}\right\}$ and $I_{2}:=\left\{n^{\alpha-1}<|y| \leq \pi\right\}$, where $\alpha>0$ is such that $q n^{\alpha-1} \rightarrow 0$. Such $\alpha$ exists because $q$ satisfies $(2.3)$. Since $|\widehat{\psi}(x)|^{2}=\left(2 \pi / \sigma^{2}\right) f(x) \leq$ $C|x|^{-2 d}$, we obtain that

$$
i_{n} \leq C q^{2-4 d} n^{-2}\left(i_{n, 1}+i_{n, 2}\right)
$$


where

$i_{n, j}:=\int_{-\pi}^{\pi} \int_{-\pi}^{\pi} \mathrm{d} x_{1} \mathrm{~d} x_{2}\left|x_{1}\right|^{-2 d}\left|x_{2}\right|^{-2 d}\left|\int_{I_{j}} \mathrm{~d} y \beta(y) D_{n}\left(x_{1}+y\right) D_{n}\left(x_{2}-y\right)\right|^{2}, \quad j=1,2$.

We show that

$$
i_{n, j}=o\left(n^{4 d}\right), \quad n \rightarrow \infty, \quad j=1,2,
$$

which, together with (A.52), proves (A.48). To work out $i_{n, 1}$, note that if $x \in I_{1}$, then $|q x| \leq q n^{\alpha-1} \rightarrow 0$, and therefore

$$
\sup _{x \in I_{1}} \beta(x)=\sup _{x \in I_{1}}\left|\left(\frac{\sin (q x / 2)}{q \sin (x / 2)}\right)^{2}-1\right|=o(1) .
$$

Applying (A.29) of Lemma A.3, we see that

$$
\begin{aligned}
\left|\int_{I_{1}} \mathrm{~d} y \beta(\lambda) D_{n}\left(x_{1}+y\right) D_{n}\left(x_{2}-y\right)\right|^{2} & =o(1)\left|\int_{I_{1}} \mathrm{~d} y\right| D_{n}\left(x_{1}+y\right) D_{n}\left(x_{2}-y\right)||^{2} \\
& =o(1) n^{2}\left(1+\left|n\left(x_{1}+x_{2}\right)\right|\right)^{-2+2 \epsilon^{\prime}}
\end{aligned}
$$

with some $0<\epsilon^{\prime}<1 / 4$. Then,

$$
\begin{aligned}
i_{n, 1} & =o(1) \int_{-\pi}^{\pi} \int_{-\pi}^{\pi}\left|x_{1}\right|^{-2 d}\left|x_{2}\right|^{-2 d} n^{2}\left(1+\left|n\left(x_{1}+x_{2}\right)\right|\right)^{-2+2 \epsilon^{\prime}} \mathrm{d} x_{1} \mathrm{~d} x_{2} \\
& =o(1) n^{4 d} \int_{-\infty}^{\infty} \int_{-\infty}^{\infty}\left|x_{1}\right|^{-2 d}\left|x_{2}\right|^{-2 d}\left(1+\left|x_{1}+x_{2}\right|\right)^{-2+2 \epsilon^{\prime}} \mathrm{d} x_{1} \mathrm{~d} x_{2}=o\left(n^{4 d}\right),
\end{aligned}
$$

since the last integral is finite when $4 d>1$.

To work out $i_{n, 2}$, recall (A.16) and write

$$
i_{n, 2}=\left.\left.\int_{I_{2}} \int_{I_{2}} \mathrm{~d} y_{1} \mathrm{~d} y_{2} \beta\left(y_{1}\right) \beta\left(y_{2}\right)\left|\int_{-\pi}^{\pi} \mathrm{d} x\right| x\right|^{-2 d} D_{n}\left(y_{1}+x\right) D_{n}\left(y_{2}-x\right)\right|^{2} .
$$

Since $\beta(y) \leq C$, we can bound $i_{n, 2} \leq C\left(p_{n, 1}+p_{n, 2}\right)$, where

$$
p_{n, i}=\left.\left.\int_{I_{2}} \int_{I_{2}} \mathrm{~d} y_{1} \mathrm{~d} y_{2}\left|\int_{J_{i}} \mathrm{~d} x\right| x\right|^{-2 d} D_{n}\left(y_{1}+x\right) D_{n}\left(y_{2}-x\right)\right|^{2}, \quad i=1,2
$$

and $J_{1}:=\left\{n^{\alpha / 2-1} \leq|x| \leq \pi\right\}, J_{2}:=\left\{|x| \leq n^{\alpha / 2-1}\right\}$.

Now,

$$
\begin{aligned}
p_{n, 1} & \leq\left.\left. C \int_{-\pi}^{\pi} \int_{-\pi}^{\pi} \mathrm{d} y_{1} \mathrm{~d} y_{2}\left|\int_{J_{1}} \mathrm{~d} x\right| x\right|^{-2 d} D_{n}\left(y_{1}+x\right) D_{n}\left(y_{2}-x\right)\right|^{2} \\
& \leq C \int_{J_{1}} \int_{J_{1}}\left|x_{1}\right|^{-2 d}\left|x_{2}\right|^{-2 d}\left|D_{n}\left(x_{1}+x_{2}\right)\right|^{2} \mathrm{~d} x_{1} \mathrm{~d} x_{2} \\
& \leq C\left(\int_{n^{\alpha / 2-1} \leq\left|x_{1}\right|,\left|x_{2}\right| \leq 1}\left|x_{1}\right|^{-2 d}\left|x_{2}\right|^{-2 d}\left|D_{n}\left(x_{1}+x_{2}\right)\right|^{2} \mathrm{~d} x_{1} \mathrm{~d} x_{2}\right.
\end{aligned}
$$




$$
\left.+\int_{1 \leq\left|x_{1}\right| \leq \pi} \int_{-\pi}^{\pi}\left|x_{2}\right|^{-2 d}\left|D_{n}\left(x_{1}+x_{2}\right)\right|^{2} \mathrm{~d} x_{1} \mathrm{~d} x_{2}\right)=: C\left(r_{n}^{\prime}+r_{n}^{\prime \prime}\right) .
$$

Using the bound (A.17), we have

$$
\begin{aligned}
r_{n}^{\prime} & \leq C \int_{n^{\alpha / 2-1} \leq\left|x_{1}\right|,\left|x_{2}\right| \leq 1}\left|x_{1}\right|^{-2 d}\left|x_{2}\right|^{-2 d} n^{2}\left(1+n\left|x_{1}+x_{2}\right|\right)^{-2} \mathrm{~d} x_{1} \mathrm{~d} x_{2} \\
& \leq C n^{4 d} \int_{n^{\alpha / 2} \leq\left|x_{1}\right|,\left|x_{2}\right|<\infty}\left|x_{1}\right|^{-2 d}\left|x_{2}\right|^{-2 d}\left(1+\left|x_{1}+x_{2}\right|\right)^{-2} \mathrm{~d} x_{1} \mathrm{~d} x_{2}=o\left(n^{4 d}\right),
\end{aligned}
$$

since $n^{\alpha} \rightarrow \infty$ and the last integral is finite when $4 d>1$.

On the other hand,

$$
r_{n}^{\prime \prime} \leq C \int_{0 \leq\left|x_{2}\right| \leq \pi}\left|x_{2}\right|^{-2 d} \mathrm{~d} x_{2} \int_{0 \leq|u| \leq 2 \pi}\left|D_{n}(u)\right|^{2} \mathrm{~d} u=O(n)=o\left(n^{4 d}\right),
$$

since $4 d>1$. Thus $p_{n, 1}=o\left(n^{4 d}\right)$.

It remains to show that $p_{n, 2}=o\left(n^{4 d}\right)$. By (A.17),

$$
\begin{aligned}
p_{n, 2} & \leq\left.\left. C \int_{n^{\alpha-1} \leq\left|y_{1}\right|,\left|y_{2}\right| \leq \pi} \mathrm{d} y_{1} \mathrm{~d} y_{2}\left|\int_{|x| \leq n^{\alpha / 2-1}} \mathrm{~d} x\right| x\right|^{-2 d} n\left(1+n\left|y_{1}+x\right|\right)^{-1} n\left(1+n\left|y_{2}-x\right|\right)^{-1}\right|^{2} \\
& \leq\left.\left. C n^{4 d} \int_{n^{\alpha} \leq\left|y_{1}\right|,\left|y_{2}\right|<\infty} \mathrm{d} y_{1} \mathrm{~d} y_{2}\left|\int_{|x| \leq n^{\alpha / 2}} \mathrm{~d} x\right| x\right|^{-2 d}\left(1+\left|y_{1}+x\right|\right)^{-1}\left(1+\left|y_{2}-x\right|\right)^{-1}\right|^{2} \\
& =: C n^{4 d} t_{n} .
\end{aligned}
$$

It suffices to show that $t_{n} \rightarrow 0$. As $n \rightarrow \infty$, in the integral above we can apply the bound $\left|y_{i} \pm x\right| \geq\left|y_{i}\right|-|x| \geq\left|y_{i}\right| / 2, i=1,2$. Therefore

$$
\begin{aligned}
& \int_{|x| \leq n^{\alpha / 2}} \mathrm{~d} x|x|^{-2 d}\left(1+\left|y_{1}+x\right|\right)^{-1}\left(1+\left|y_{2}+x\right|\right)^{-1} \\
\leq & C\left(1+\left|y_{1}\right|\right)^{-1 / 2-d / 2}\left(1+\left|y_{2}\right|\right)^{-1 / 2-d / 2} \\
& \times \int_{|x| \leq n^{\alpha / 2}} \mathrm{~d} x|x|^{-2 d}\left(\left(1+\left|y_{1}+x\right|\right)^{-1+d}+\left(1+\left|y_{2}+x\right|\right)^{-1+d}\right) \\
\leq & C\left(1+\left|y_{1}\right|\right)^{-1 / 2-d / 2}\left(1+\left|y_{2}\right|\right)^{-1 / 2-d / 2},
\end{aligned}
$$

for $y_{1}, y_{2}$. Thus,

$$
t_{n} \leq C \int_{n^{\alpha} \leq\left|y_{1}\right|,\left|y_{2}\right| \leq \infty}\left(1+\left|y_{1}\right|\right)^{-1-d}\left(1+\left|y_{2}\right|\right)^{-1-d} \mathrm{~d} y_{1} \mathrm{~d} y_{2} \rightarrow 0,
$$

to complete the proof of (A.53) and (A.48).

Proof of (A.49). By (A.47), $U_{n}=(q / n)^{1-2 d} n^{-2 d} \sum_{t=1}^{n} X_{t}^{2}$. Therefore,

$$
(n / q)^{1-2 d}\left(U_{n}-\mathrm{E}\left(U_{n}\right)\right)=n^{-2 d} \sum_{t=1}^{n}\left(X_{t}^{2}-\mathrm{E}\left(X_{t}^{2}\right)\right) .
$$


By Theorem 2.1 in Giraitis, Taqqu and Terrin (1998),

$$
n^{-2 d} \sum_{t=1}^{n}\left(X_{t}^{2}-\mathrm{E}\left(X_{t}^{2}\right)\right) \stackrel{d}{\longrightarrow} b_{0} \int_{\mathbb{R}^{2}}^{\prime \prime} \Psi_{0}\left(x_{1}, x_{2}\right)\left|x_{1}\right|^{-d}\left|x_{2}\right|^{-d} Z\left(\mathrm{~d} x_{1}\right) Z\left(\mathrm{~d} x_{2}\right),
$$

with

$$
\Psi_{0}\left(x_{1}, x_{2}\right):=\int_{\mathbb{R}} \frac{\mathrm{e}^{\mathrm{i}\left(x_{1}+u\right)}-1}{\mathrm{i}\left(x_{1}+u\right)} \frac{\mathrm{e}^{\mathrm{i}\left(x_{2}-u\right)}-1}{\mathrm{i}\left(x_{1}-u\right)} \mathrm{d} u=2 \pi \frac{\mathrm{e}^{\mathrm{i}\left(x_{1}+x_{2}\right)}-1}{\mathrm{i}\left(x_{1}+x_{2}\right)} .
$$

Hence,

$$
(n / q)^{1-2 d}\left(U_{n}-\mathrm{E}\left(U_{n}\right)\right) \stackrel{d}{\longrightarrow} 2 \pi b_{0} R(d),
$$

to prove (A.49).

Proof of (A.50). By (2.1),

$$
\begin{aligned}
\mathrm{E}\left(\widetilde{s}_{q}^{2}(d)\right) & =\int_{-\pi}^{\pi} b_{n}(\lambda) \mathrm{E}\left(I_{n}(\lambda)\right) \mathrm{d} \lambda=(2 \pi n)^{-1} \int_{-\pi}^{\pi} \int_{-\pi}^{\pi} b_{n}(\lambda) f(x)\left|D_{n}(\lambda+x)\right|^{2} \mathrm{~d} \lambda \mathrm{d} x \\
& =(2 \pi n)^{-1} \int_{-\pi}^{\pi}\left(\frac{\sin (u n / 2)}{\sin (u / 2)}\right)^{2} H_{n}(u) \mathrm{d} u,
\end{aligned}
$$

where $H_{n}(u)$ is defined in (A.23). Then,

$$
\begin{aligned}
\mathrm{E}\left(\widetilde{s}_{q}^{2}(d)\right)= & (2 \pi n)^{-1} \int_{-\pi}^{\pi}\left|D_{n}(u)\right|^{2} H_{n}(0) \mathrm{d} u \\
& +(2 \pi n)^{-1} \int_{-\pi}^{\pi}\left|D_{n}(u)\right|^{2}\left(H_{n}(u)-H_{n}(0)\right) \mathrm{d} u=: v_{n, 1}+v_{n, 2} .
\end{aligned}
$$

By Proposition A.2,

$$
v_{n, 1}=H_{n}(0)=s_{X}^{2}+q^{-1-2 d} B+o\left(q^{-1-2 d}\right) .
$$

To prove (A.50), it remains to show that

$$
v_{n, 2}=o\left(q^{-1-2 d}\right)+o\left((q / n)^{1-2 d}\right) .
$$

Set $p_{n}:=(n / q)^{\epsilon}$ where $\epsilon>0$ is a small number satisfying (A.18). By (A.24),

$$
\begin{aligned}
& n^{-1} \int_{|u| \leq p_{n}^{d} / n}\left|D_{n}(u)\right|^{2}\left|H_{n}(u)-H_{n}(0)\right| \mathrm{d} u \\
= & {\left[o\left((q / n)^{1-2 d}\right)+o\left(q^{-1-2 d}\right)\right] n^{-1} \int_{|u| \leq \pi}\left|D_{n}(u)\right|^{2} \mathrm{~d} u=\left[o\left((q / n)^{1-2 d}\right)+o\left(q^{-1-2 d}\right)\right], }
\end{aligned}
$$

whereas by (A.25), $\left|H_{n}(u)-H_{n}(0)\right| \leq C(|u| q)^{1-2 d}$, and therefore

$$
n^{-1} \int_{p_{n}^{d} / n \leq|u| \leq \pi}\left|\sum_{t=1}^{n} \mathrm{e}^{\mathrm{i} t u}\right|^{2}\left|H_{n}(u)-H_{n}(0)\right| \mathrm{d} u
$$




$$
\begin{aligned}
& \leq C q^{1-2 d} n^{-1} \int_{p_{n}^{d} / n \leq|u| \leq \pi}\left(\frac{\sin (u n / 2)}{u / 2}\right)^{2}|u|^{1-2 d} \mathrm{~d} u \\
& \leq C(q / n)^{1-2 d} \int_{p_{n}^{d} \leq|u| \leq \infty}\left(\frac{\sin (u / 2)}{u / 2}\right)^{2}|u|^{1-2 d} \mathrm{~d} u=o\left((q / n)^{1-2 d}\right),
\end{aligned}
$$

since $p_{n}^{d} \rightarrow \infty$, to prove (A.54).

\section{Acknowledgments}

We are grateful for the feedback of seminar participants at GREQAM, Imperial, Liverpool, Oxford, Tilburg, and the 2006 International Vilnius Conference on Probability Theory and Mathematical Statistics. We also thank two referees and Peter Robinson for their comments. This research is supported by ESRC grants R000239538, RES000230176, and RES062230790.

\section{References}

Abadir, K.M., Distaso, W., Giraitis, L., 2007, Nonstationarity-extended local Whittle estimation. Journal of Econometrics, 141, 1353-1384.

Abadir, K. M., Talmain, G., 2002, Aggregation, persistence and volatility in a macro model. Review of Economic Studies 69, 749-779.

Abadir, K. M., Taylor, A. M. R., 1999, On the definitions of (co-)integration. Journal of Time Series Analysis 20, 129-137.

Andrews, D. W. K., Monahan, J. C., 1992, An improved heteroskedasticity and autocorrelation consistent covariance matrix estimator. Econometrica 60, 953966.

Baillie, R. T., Bollerslev, T., 1994, Cointegration, fractional cointegration, and exchange rate dynamics. Journal of Finance 49, 737-745.

Bhansali, R. J., Giraitis, L., Kokoszka, P., 2007, Decomposition and asymptotic properties of quadratic forms in linear variables. Stochastic Processes and their Applications 117, 71-95.

Cavaliere, G., 2001, Testing the unit root hypothesis using generalized range statistics. Econometrics Journal 4, 70-88.

Chambers, M. J., 1998, Long memory and aggregation in macroeconomic time series. International Economic Review 39, 1053-1072. 
Dalla, V., Giraitis, L., Hidalgo, J., 2006, Consistent estimation of the memory parameter for nonlinear time series. Journal of Time Series Analysis 27, 211251.

Diebold, F. X., Rudebusch, G. D., 1989, Long memory and persistence in aggregate output. Journal of Monetary Economics 24, 189-209.

Gil-Alaña, L. A., Robinson, P. M., 1997, Testing of unit root and other nonstationary hypotheses in macroeconomic time series. Journal of Econometrics 80, $241-268$.

Giraitis, L., Kokoszka, P., Leipus, R., Tessière, G., 2003, Rescaled variance and related tests for long memory in volatility and levels. Journal of Econometrics $112,265-294$.

Giraitis, L., Taqqu, M. S., Terrin, N., 1998, Limit theorems for bivariate Appell polynomials. Part: II. Non-Central Limit Theorems. Probability Theory and Related Fields 110, 333-367.

Hannan, E. J., 1957, The variance of the mean of a stationary process. Journal of the Royal Statistical Society B 19, 282-285.

Jowett, G. H., 1955, The comparison of means of sets of observations from sections of independent stochastic series. Journal of the Royal Statistical Society B 17, 208-227.

Newey, W. K., West, K. D., 1987, A simple, positive semi-definite, heteroskedasticity and autocorrelation consistent covariance matrix. Econometrica 55, 703708 .

Robinson, P. M., 1995a, Log-periodogram regression of time series with long range dependence. Annals of Statistics 23, 1048-1072.

Robinson, P. M., 1995b, Gaussian semiparametric estimation of long range dependence. Annals of Statistics 23, 1630-1661.

Robinson, P. M., 1997, Large-sample inference for nonparametric regression with dependent errors. Annals of Statistics 25, 2054-2083.

Robinson, P. M., 2005, Robust covariance matrix estimation: HAC estimates with long memory/antipersistence correction. Econometric Theory 21, 171-180. 
Velasco, C., Robinson, P. M., 2001, Edgeworth expansions for spectral density estimates and studentized sample mean. Econometric Theory 17, 497-539.

White, H., 1980, A heteroskedasticity-consistent covariance matrix estimator and a direct test for heteroskedasticity. Econometrica 48, 817-838. 
Table 1: MSE of HAC estimator $\bar{s}_{q}^{2}(\widehat{d})$.

\begin{tabular}{|c|c|c|c|c|c|c|c|c|c|}
\hline$d$ & \multicolumn{3}{|c|}{$n=250$} & \multicolumn{3}{|c|}{$n=500$} & \multicolumn{3}{|c|}{$n=1000$} \\
\hline \multirow{8}{*}{$\begin{aligned} d & =-0.4 \\
d & =-0.2 \\
d & =0 \\
d & =0.2 \\
d & =0.4\end{aligned}$} & \multirow{2}{*}{\multicolumn{3}{|c|}{ 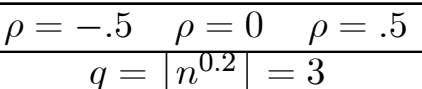 }} & \multicolumn{3}{|c|}{$\rho=-.5 \quad \rho=0 \quad \rho=.5$} & \multicolumn{3}{|c|}{$\rho=-.5 \quad \rho=0 \quad \rho=.5$} \\
\hline & & & & \multirow{2}{*}{$\frac{q=}{54}$} & \multicolumn{2}{|c|}{$n^{0.2}=3$} & \multicolumn{3}{|c|}{\begin{tabular}{l|l|l}
$q=$ & $n^{0.2}$ & $=3$
\end{tabular}} \\
\hline & 6.138 & $\stackrel{\rfloor}{4.992}$ & 2.642 & & 5.033 & 1.983 & 6.047 & 4.86 & 1.469 \\
\hline & 0.363 & \multicolumn{2}{|c|}{$0.140 \quad 0.422$} & 0.379 & 0.126 & 0.740 & 0.395 & 0.105 & 0.954 \\
\hline & 0.149 & 0.051 & 1.208 & 0.158 & 0.039 & 1.657 & 0.162 & 0.020 & 2.000 \\
\hline & 0.188 & 0.053 & 3.192 & 0.176 & 0.037 & 3.387 & 0.175 & 0.033 & 4.285 \\
\hline & 1.477 & \multicolumn{2}{|c|}{0.305} & 1.348 & 0.214 & 14.489 & 1.275 & 0.124 & 16.188 \\
\hline & $q=$ & $n^{0.3}$ & $=5$ & $q=$ & $n^{0.3}$ & $=6$ & $q=$ & $n^{0.3}$ & $=7$ \\
\hline$d=-0.4$ & 5.749 & 4.319 & 2.048 & 5.442 & 4.044 & 1.082 & 5.244 & 3.65 & 0.722 \\
\hline$d=-$ & 0.358 & 0.186 & 0.723 & 0.357 & 0.146 & 1.783 & 0.391 & 0.11 & 2.595 \\
\hline$d=0$ & 0.176 & 0.080 & 1.173 & 0.200 & 0.061 & 2.001 & 0.210 & 0.05 & 2.784 \\
\hline$d=$ & 0.225 & 0.061 & 2.990 & 0.243 & 0.051 & 2.636 & 0.254 & 0.03 & 3.586 \\
\hline$d=0.4$ & 1.681 & 0.493 & 7.439 & 1.669 & 0.397 & 9.628 & 1.625 & 0.32 & 12.018 \\
\hline & $q=$ & $n^{0.4}$ & $=9$ & $q=$ & $n^{0.4}$ & $=12$ & $q=$ & $n^{0.4}$ & $=15$ \\
\hline$d=-0.4$ & 5.472 & 3.881 & 2.029 & 5.211 & 3.356 & 1.372 & 5.030 & 2.87 & 1.715 \\
\hline$d=-$ & 88 & 0.234 & 0.978 & 02 & 0.176 & 2.194 & .432 & 0.15 & 4.053 \\
\hline$d=0$ & 0.200 & 0.109 & 0.908 & 0.236 & 0.089 & 2.006 & .252 & 0.07 & 2.929 \\
\hline$d=$ & 0.285 & 0.076 & 2.702 & 0.293 & 0.053 & 1.902 & 0.296 & 0.03 & 2.632 \\
\hline$d=0.4$ & 1.914 & 0.726 & 6.205 & 1.906 & 0.664 & 6.506 & 1.883 & 0.56 & 7.444 \\
\hline & $q=$ & $\left.n^{0.5}\right]=$ & $=15$ & $q=$ & $\left.n^{0.5}\right]=$ & $=22$ & $q=$ & $n^{0.5}$ & $=31$ \\
\hline$d=$ & 5.298 & 3.547 & 2.023 & 187 & 2.909 & 1.8 & $\overline{043}$ & 2.37 & 2.962 \\
\hline$d=$ & 0.424 & 0.373 & 0.910 & 0.436 & 0.293 & 2.652 & 0.477 & 0.21 & 3.740 \\
\hline$d=0$ & 0.230 & 0.167 & 0.853 & 0.256 & 0.118 & 1.620 & 0.269 & 0.06 & 2.702 \\
\hline$d=0.2$ & 0.307 & 0.116 & 2.716 & 0.334 & 0.075 & 1.410 & 0.334 & 0.05 & 1.754 \\
\hline$d=$ & 2.067 & 1.007 & 3.617 & 2.096 & 0.892 & 4.817 & 2.094 & 0.76 & 5.556 \\
\hline & $q=$ & $n^{0.6}$ & $=27$ & $q=$ & \begin{tabular}{l|l}
$n^{0.6}$ & $=$
\end{tabular} & $=41$ & $q=$ & $n^{0.6}$ & $=63$ \\
\hline$d=-0.4$ & 5.207 & 3.223 & 2.593 & 5.017 & 2.775 & 2.316 & 4.919 & 2.22 & 4.341 \\
\hline$d=-$ & 0.436 & 0.368 & 0.995 & 0.467 & 0.308 & 1.883 & 0.515 & 0.30 & 3.589 \\
\hline$d=$ & 0.268 & 0.209 & 0.513 & 0.302 & 0.128 & 1.164 & 0.294 & 0.10 & 1.991 \\
\hline$d=$ & 0.383 & 0.155 & 1.774 & 0.373 & 0.134 & 0.842 & 0.384 & 0.09 & 1.068 \\
\hline$d=$ & 2.333 & 1.379 & 3.205 & 2.362 & 1.236 & 3.138 & 2.324 & 1.18 & 3.806 \\
\hline & $q=$ & $\left.n^{0.7}\right]=$ & $=47$ & $q=$ & $n^{0.7}=$ & $=77$ & $q=$ & $n^{0.7}$ & $=125$ \\
\hline$d=$ & 5.113 & 3.228 & 2.958 & 5.022 & 2.512 & 3.170 & 4.845 & 1.94 & 6.103 \\
\hline$d=-0.2$ & 0.511 & 0.463 & 1.077 & 0.528 & 0.499 & 1.764 & 0.543 & 0.31 & 3.416 \\
\hline & 0.311 & 0.328 & 0.535 & 0.336 & 0.180 & 0.844 & 0.343 & 0.13 & 1.552 \\
\hline$d=0.2$ & 0.452 & 0.245 & 1.704 & 0.427 & 0.223 & 0.646 & 0.452 & 0.17 & 0.786 \\
\hline$d=0.4$ & 2.598 & 1.762 & 2.038 & 2.593 & 1.672 & 2.255 & 2.532 & 1.61 & 2.755 \\
\hline & $q=$ & $\left.n^{0.8}\right]=$ & $=82$ & $q=$ & $n^{0.8}=$ & $=144$ & $q=$ & $n^{0.8}$ & $=251$ \\
\hline$d=-$ & 4.986 & 3.072 & 4.400 & 4.847 & 2.687 & 4.829 & 4.796 & 2.10 & 8.090 \\
\hline$d=-0$ & 0.632 & 0.857 & 0.954 & 0.581 & 0.569 & 2.606 & 0.588 & 0.40 & 3.847 \\
\hline$d=0$ & 0.406 & 0.434 & 0.394 & 0.423 & 0.307 & 0.748 & 0.415 & 0.25 & 1.221 \\
\hline & 0.548 & 0.373 & 1.063 & 0.559 & 0.338 & 0.440 & 0.543 & 0.31 & 0.570 \\
\hline$d=0.4$ & 2.941 & 2.229 & 2.006 & 2.878 & 2.264 & 2.290 & 2.913 & 2.15 & 1.976 \\
\hline
\end{tabular}


Table 2: MSE of HAC estimator $\bar{s}_{q}^{2}(\widehat{d})$ when $q$ is chosen according to (2.14).

\begin{tabular}{|l|ccc|ccc|ccc|}
\hline$d$ & \multicolumn{3}{|c|}{$n=250$} & \multicolumn{3}{c|}{$n=500$} & \multicolumn{3}{c|}{$n=1000$} \\
\hline & $\rho=-.5$ & $\rho=0$ & $\rho=.5$ & $\rho=-.5$ & $\rho=0$ & $\rho=.5$ & $\rho=-.5$ & $\rho=0$ & $\rho=.5$ \\
\cline { 2 - 10 }$d=-0.4$ & 5.028 & 3.101 & 2.178 & 4.864 & 2.516 & 3.300 & 4.721 & 2.046 & 5.409 \\
$d=-0.2$ & 0.397 & 0.489 & 1.133 & 0.457 & 0.306 & 2.068 & 0.439 & 0.258 & 3.808 \\
$d=0$ & 0.189 & 0.122 & 1.236 & 0.219 & 0.117 & 2.064 & 0.242 & 0.051 & 2.926 \\
$d=0.2$ & 0.211 & 0.065 & 3.495 & 0.224 & 0.049 & 3.231 & 0.241 & 0.029 & 4.093 \\
$d=0.4$ & 1.384 & 0.219 & 10.275 & 1.296 & 0.164 & 11.480 & 1.218 & 0.101 & 12.527 \\
\hline
\end{tabular}

Table 3: MSE of MAC estimator $\widehat{s}_{m}^{2}(\widehat{d})$.

\begin{tabular}{|c|c|c|c|c|c|c|c|c|c|}
\hline$d$ & \multicolumn{3}{|c|}{$n=250$} & \multicolumn{3}{|c|}{$n=500$} & \multicolumn{3}{|c|}{$n=1000$} \\
\hline \multirow{8}{*}{$\begin{array}{l}d=-0.4 \\
d=-0.2 \\
d=0 \\
d=0.2 \\
d=0.4\end{array}$} & \multirow{2}{*}{$\begin{array}{r}\rho=-.5 \\
m=\end{array}$} & \multirow{2}{*}{\multicolumn{2}{|c|}{\begin{tabular}{l}
\multicolumn{2}{c}{$\rho=0 \quad \rho=.5$} \\
$n^{0.5}=15$
\end{tabular}}} & \multirow{2}{*}{$\begin{array}{r}\rho=-.5 \\
m=\end{array}$} & \multirow{2}{*}{\multicolumn{2}{|c|}{\begin{tabular}{l|l}
$\rho=0 \quad$ & $\rho=.5$ \\
$n^{0.5}$ & $=22$
\end{tabular}}} & \multirow{2}{*}{$\frac{\rho=-.5}{m=}$} & \multicolumn{2}{|c|}{$\rho=0 \quad \rho=.5$} \\
\hline & & & & & & & & $n^{0.5}$ & $=31$ \\
\hline & 5.097 & 2.695 & 6.667 & 5.055 & 2.05 & 16.202 & 4.861 & 1.87 & 25.473 \\
\hline & 0.650 & 1.952 & 2.289 & 0.557 & 1.20 & 6.749 & 0.548 & 0.53 & 5.767 \\
\hline & 0.256 & 0.249 & 1.948 & 0.260 & 0.15 & 2.895 & 0.272 & 0.08 & 3.988 \\
\hline & 0.235 & 0.101 & 7.623 & 0.262 & 0.05 & 5.787 & 0.277 & 0.03 & 5.144 \\
\hline & 1.127 & 0.317 & 32.052 & 1.110 & 0.24 & 35.765 & 1.089 & 0.19 & 36.869 \\
\hline & $m=$ & $n^{0.6}$ & $=27$ & $m=$ & $n^{0.6}$ & $=41$ & $m=$ & $n^{0.6}$ & $=63$ \\
\hline$d=-0.4$ & 4.814 & 2.446 & 7.032 & 4.632 & 2.26 & 15.770 & 4.641 & 1.89 & 28.134 \\
\hline$d=-$ & 0.619 & 1.182 & 2.626 & 19 & 0.65 & 4.186 & 52 & 0.49 & 6.278 \\
\hline$d=0$ & 0.260 & 0.416 & 1.781 & 0.276 & 0.13 & 2.866 & 0.271 & 0.11 & 3.973 \\
\hline$d=0.2$ & 0.246 & 0.077 & 6.269 & 0.260 & 0.05 & 5.616 & 0.280 & 0.03 & 5.269 \\
\hline$d=0.4$ & 1.141 & 0.264 & 22.630 & 1.195 & 0.23 & 26.383 & 1.134 & 0.15 & 31.099 \\
\hline & $m=$ & $n^{0.7}$ & $=47$ & $m=$ & $n^{0.7}$ & $=77$ & $m=$ & $n^{0.7}$ & $=125$ \\
\hline$d=-0.4$ & 4.215 & 1.987 & 5.260 & 4.460 & 1.91 & 9.449 & 4.365 & 1.66 & 19.986 \\
\hline$d=-0.2$ & 0.497 & 0.580 & 1.174 & 0.507 & 0.89 & 2.453 & 0.510 & 0.26 & 3.939 \\
\hline$d=0$ & 0.204 & 0.201 & 1.032 & 0.241 & 0.08 & 1.644 & 0.263 & 0.05 & 2.605 \\
\hline$d=0.2$ & 0.203 & 0.057 & 4.138 & 0.236 & 0.02 & 3.575 & 0.266 & 0.01 & 3.827 \\
\hline$d=0.4$ & 1.124 & 0.259 & 9.757 & 1.157 & 0.19 & 14.549 & 1.136 & 0.13 & 19.078 \\
\hline & $m=$ & $n^{0.8}$ & $=82$ & $m=$ & $n^{0.8}$ & $=144$ & $m=$ & $n^{0.8}$ & $=251$ \\
\hline$d=-0.4$ & 2.896 & 1.603 & 3.176 & 3.138 & 1.47 & 3.044 & 3.454 & 1.34 & 5.342 \\
\hline$d=-0.2$ & 0.316 & 0.773 & 0.361 & 0.329 & 0.21 & 0.900 & 0.364 & 0.11 & 1.133 \\
\hline$d=$ & 0.102 & 0.065 & 0.327 & 0.150 & 0.07 & 0.517 & 0.186 & 0.02 & 0.809 \\
\hline$d=0.2$ & 0.099 & 0.075 & 2.133 & 0.140 & 0.01 & 1.682 & 0.185 & 0.01 & 1.381 \\
\hline$d=0.4$ & 0.696 & 0.264 & 3.188 & 0.769 & 0.23 & 4.667 & 0.840 & 0.20 & 6.192 \\
\hline
\end{tabular}


Table 4: Coverage probabilities for $\mu$ based on the t-ratio (4.1) and the HAC estimator $\bar{s}_{q}^{2}(\widehat{d})$ with $q$ chosen according to $(2.14)$.

\begin{tabular}{|l|ccc|ccc|ccc|}
\hline$d$ & \multicolumn{3}{|c|}{$n=250$} & \multicolumn{3}{|c|}{$n=500$} & \multicolumn{3}{c|}{$n=1000$} \\
\hline & $\rho=-.5$ & $\rho=0$ & $\rho=.5$ & $\rho=-.5$ & $\rho=0$ & $\rho=.5$ & $\rho=-.5$ & $\rho=0$ & $\rho=.5$ \\
\cline { 2 - 10 }$d=-0.4$ & 0.984 & 0.956 & 0.972 & 0.970 & 0.958 & 0.942 & 0.984 & 0.948 & 0.950 \\
$d=-0.2$ & 0.916 & 0.912 & 0.966 & 0.932 & 0.918 & 0.958 & 0.928 & 0.922 & 0.956 \\
$d=0$ & 0.890 & 0.880 & 0.964 & 0.914 & 0.896 & 0.962 & 0.922 & 0.930 & 0.958 \\
$d=0.2$ & 0.904 & 0.888 & 0.968 & 0.890 & 0.878 & 0.970 & 0.926 & 0.910 & 0.970 \\
$d=0.4$ & 0.830 & 0.802 & 0.862 & 0.822 & 0.830 & 0.870 & 0.884 & 0.870 & 0.898 \\
\hline
\end{tabular}

Table 5: Coverage probabilities for $\mu$ based on the t-ratio (4.1) and the MAC estimator $\widehat{s}_{m}^{2}(\widehat{d})$.

\begin{tabular}{|c|c|c|c|c|c|c|c|c|c|}
\hline \multirow{9}{*}{$\begin{array}{l}d=-0.4 \\
d=-0.2 \\
d=0 \\
d=0.2 \\
d=0.4\end{array}$} & \multicolumn{3}{|c|}{$n=250$} & \multicolumn{3}{|c|}{$n=500$} & \multicolumn{3}{|c|}{$n=1000$} \\
\hline & \multirow{2}{*}{\multicolumn{3}{|c|}{ 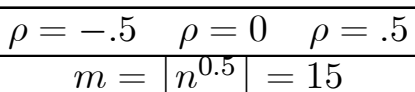 }} & \multicolumn{3}{|c|}{$\rho=-.5 \quad \rho=0 \quad \rho=.5$} & \multicolumn{3}{|c|}{$\rho=-.5 \quad \rho=0 \quad \rho=.5$} \\
\hline & & & & $m=$ & $n^{0.5}$ & $=22$ & $m=$ & $n^{0.5}$ & $=31$ \\
\hline & 0.972 & 0.984 & 0.988 & 0.964 & 0.984 & 0.990 & 0.966 & 0.97 & 0.992 \\
\hline & 0.924 & 0.922 & 0.980 & 0.948 & 0.948 & 0.968 & 0.936 & 0.93 & 0.968 \\
\hline & 0.866 & 0.890 & 0.970 & 0.884 & 0.916 & 0.974 & 0.892 & 0.93 & 0.964 \\
\hline & 0.866 & 0.896 & 0.960 & 0.884 & 0.924 & 0.982 & 0.908 & 0.91 & 0.968 \\
\hline & 0.792 & 0.862 & 0.904 & 0.822 & 0.858 & 0.934 & 0.888 & 0.88 & 0.932 \\
\hline & $m=$ & $n^{0.6}$ & $=27$ & $m=$ & $n^{0.6}$ & $=41$ & $m=$ & $n^{0.6}$ & $=63$ \\
\hline$d=-0.4$ & $\overline{0.962}$ & 0.978 & 0.986 & 0.968 & 0.984 & $\overline{0.992}$ & $\overline{0.962}$ & 0.98 & 0.980 \\
\hline$d=-0.2$ & 0.912 & 0.940 & 0.970 & 0.924 & 0.948 & 0.966 & 0.922 & 0.92 & 0.960 \\
\hline$d=0$ & 0.878 & 0.898 & 0.970 & 0.894 & 0.912 & 0.962 & 0.912 & 0.92 & 0.972 \\
\hline$d=0.2$ & 0.908 & 0.892 & 0.968 & 0.886 & 0.912 & 0.974 & 0.914 & 0.91 & 0.984 \\
\hline$d=0.4$ & 0.786 & 0.838 & 0.916 & 0.838 & 0.856 & 0.934 & 0.844 & 0.86 & 0.932 \\
\hline & $m=$ & $n^{0.7}$ & $=47$ & $m=$ & $n^{0.7}$ & $=77$ & $m=$ & $n^{0.7}$ & $=125$ \\
\hline$d=-0.4$ & 0.970 & 0.984 & 0.986 & 0.982 & 0.972 & 0.982 & 0.976 & 0.98 & 0.972 \\
\hline$d=-0.2$ & 0.938 & 0.928 & 0.978 & 0.942 & 0.940 & 0.948 & 0.912 & 0.93 & 0.970 \\
\hline$d=0$ & 0.880 & 0.904 & 0.960 & 0.894 & 0.930 & 0.966 & 0.916 & 0.92 & 0.942 \\
\hline$d=0.2$ & 0.866 & 0.870 & 0.958 & 0.880 & 0.896 & 0.958 & 0.896 & 0.94 & 0.944 \\
\hline$d=0.4$ & 0.800 & 0.806 & 0.850 & 0.876 & 0.870 & 0.920 & 0.864 & 0.86 & 0.916 \\
\hline & $m=$ & $n^{0.8}$ & $=82$ & $m=$ & $\left.n^{0.8}\right]=$ & $=144$ & $m=$ & $n^{0.8}$ & $=251$ \\
\hline$d=-0.4$ & 0.998 & 0.976 & 0.962 & 0.990 & 0.984 & 0.948 & 0.996 & 0.97 & 0.962 \\
\hline$d=-0.2$ & 0.948 & 0.910 & 0.922 & 0.936 & 0.910 & 0.934 & 0.942 & 0.92 & 0.902 \\
\hline$d=0$ & 0.890 & 0.906 & 0.950 & 0.916 & 0.890 & 0.922 & 0.936 & 0.92 & 0.936 \\
\hline$d=0.2$ & 0.874 & 0.878 & 0.920 & 0.918 & 0.922 & 0.920 & 0.938 & 0.92 & 0.942 \\
\hline$d=0.4$ & 0.866 & 0.850 & 0.792 & 0.902 & 0.862 & 0.828 & 0.912 & 0.88 & 0.852 \\
\hline
\end{tabular}

